\title{
PERIODIC AND BOUNDARY VALUE PROBLEMS FOR SECOND ORDER DIFFERENTIAL INCLUSIONS
}

\author{
MICHELA PALMUCCI \\ University of Perugia \\ Department of Mathematics, Via Vanvitelli 1 \\ Perugia 060123, Italy \\ FRANCESCA PAPALINI \\ University of Ancona \\ Department of Mathematics, Via Brecce Bianche \\ Ancona 60131, Italy
}

(Received March, 1999; Revised August, 1999)

\begin{abstract}
In this paper we study differential inclusions with boundary conditions in which the vector field $F(t, x, y)$ is a multifunction with Caratheodory type conditions. We consider, first, the case which $F$ has values in $\mathbb{R}$ and we establish the existence of extremal solutions in the order interval determined by the lower and the upper solution. Then we prove the existence of solutions for a Dirichlet problem in the case in which $F$ takes their values in a Hilbert space.
\end{abstract}

Key words: Upper Solutions, Lower Solutions, Order Interval, Truncation Map, Penalty Function, Tube Solution, Extremal Solutions.

AMS subject classifications: $34 \mathrm{~B} 15$.

\section{Introduction}

In the study of differential equations with initial or boundary conditions, different methods are used to establish the existence of solutions. One among these is the method of upper and lower solutions. It seems that probably this method appeared, for the first time, in [39] where O. Perron used the method of "sub-harmonic functions" in the potential theory. Later, in 1937, M. Nagumo introduced the method of upper and lower solutions in the study of second order differential equations with boundary conditions, in particular for Dirichlet problems. Then many authors developed and applied this method to prove the existence of solutions to problems of the form

$$
x^{\prime \prime}(t)=f\left(t, x(t), x^{\prime}(t) \text { (i.e. } f\left(t, x(t), x^{\prime}(t)\right)\right) \text { a.e. on } T=[a, b]
$$


with boundary conditions: see for example, $[12,13,15,23,33-35,37]$ in which " $f$ " is a continuous function. For the discontinuous case (at least in the time variable $t$ ) we mention, for instance, the following papers [7, 18, 25, 28].

In this context, in 1995, N. Papageorgiou-F. Papalini [38] studied equation (1.1) with Sturm-Liouville or periodic conditions and they proved the existence of extremal solutions in the order interval characterized by the lower and upper solutions, by assuming on $f$, in addition to the classical Nagumo growth condition, Caratheodorytype hypotheses. Moreover, some authors (cf. [16, 17, 30]) have also studied boundary value problems for second order differential inclusions. In 1990, M. Frigon [16] applied the method of upper and lower solutions to a boundary value problem for differential inclusions of the type:

$$
x^{\prime \prime}(t) \in F\left(t, x(t), x^{\prime}(t)\right) \text { a.e. on } T \text {. }
$$

Frigon proved (cf. [16, Theorem VI.4]) the existence of solutions in the case in which $F: T \times \mathbb{R} \times \mathbb{R} \rightarrow 2^{\mathbb{R}}$ is a particular multifunction. The author later extended the previous result (cf. [20, Theorem 5.2]) to a generic multifunction $F$.

In this paper, we first consider the differential inclusion (1.2) with Sturm-Liouvilletype or periodic conditions and we obtain a result that contains Theorem 5.2 of [20]. Specifically, under the same assumptions required by M. Frigon in Theorem 5.2 of [20], we prove the existence of extremal solutions in the order interval characterized by the lower and upper solution. Moreover, we observe that this result contains also, as a particular case, Theorem 2 of [38].

In the second part of this paper we study the following problem:

$$
\left\{\begin{array}{c}
-x^{\prime \prime}(t) \in F\left(t, x(t), x^{\prime}(t)\right) \quad \text { a.e. on } T \\
x(0)=v_{0}, x(b)=v_{1}
\end{array}\right.
$$

where $F$ is a multifunction defined on $T \times H \times H$ with values in $2^{H}$, where $H$ is a real separable Hilbert space.

M. Frigon [20], studied problem (1.3) in the case $H=\mathbb{R}^{N}$. Prior to the authors (cf. $[19,26,31,42]$ ) applied the method of upper and lower solutions to systems of differential equations by extending the notion of upper and lower solutions while $\mathrm{M}$. Frigon in [20] generalized this concept to differential inclusions by introducing the definition of "tube solution" (cf. [20, Definition 5.8]). So, under suitable conditions, Frigon proved the existence of solutions for the problem (1.3) (cf. [20, Theorem 5.9]).

In this paper, by extending in a natural way the notion of "tube solution" to Hilbert space and by assuming on " $F$ " Caratheodory-type conditions we obtain the existence of solutions for problem (1.3). Our result extends Theorem 5.9 of [20] in the sense that there exist multifunctions which satisfy our conditions but not those of the mentioned theorem of [20]).

\section{Preliminaries}

Let $X$ be a Hausdorff topological vector space, $(Y,\|\cdot\|)$ be a Banach space. If $Z$ is a nonempty subset of $Y$, we put $\|Z\|=\sup \{\|z\|: z \in Z\}$. Throughout this work, we use the following notations: 
$P_{w k c}(X)=\{A \subset X: A \neq \emptyset, A$ closed, bounded and convex $\}$

$P_{f(k c)}(X)=\{A \subset X: A \neq \emptyset, A$ closed (compact and convex) $\}$.

Let $F: Z \rightarrow 2^{X}$ be a multifunction. We denote by

$$
R(F)=\bigcup_{y \in Z} F(y) \text { and } G r F=\{(z, x) \in Z \times X: x \in F(z)\}
$$

the range and the graph of $F$ respectively. Moreover, for every subset $A$ of $X$, we put $F^{-1}(A)=\{z \in Z: F(z) \cap A \neq \emptyset\}$ and $F^{+}(A)=\{z \in Z: F(z) \subset A\}$.

$F$ is called upper semicontinuous (u.s.c.) on $Z$ if $F^{-1}(A)$ is closed, for every closed subset $A$ of $X$ (or, equivalently, if $F^{+}(A)$ is open, for every open subset $A$ of $X$ ). Another notion of upper semicontinuity is that of metric upper semicontinuity: $F$ is said to be metric upper semicontinuous (u.s.c.) ${ }_{m}$ on $Z$ if $\forall z_{0} \in Z$ and for every neighborhood $U$ of zero in $X$ there exists a neighborhood $I\left(z_{0}\right)$ of $z_{0}$, with the property

$$
F(z) \subset F\left(z_{0}\right)+U, \quad \forall z \in I\left(z_{0}\right)
$$

In general, every (u.s.c.) multifunction is also (u.s.c.) ${ }_{m}$ and the two definitions are equivalent, for instance, for compact-valued multifunctions.

The multifunction $F$ is said to have closed graph if the set $G r F$ is closed in $Z \times X$. Now we suppose that $X$ is a Banach space and $Z$ is closed in $Y$.

$F$ is said to be compact if the set $R(F)$ is relative compact in $X$; moreover, $F$ is said to have weakly sequentially closed graph if for every sequence $\left\{y_{n}\right\}_{n} \subset Z$ with $y_{n} \rightarrow y$ in $Z$ and for every sequence $\left\{x_{n}\right\}_{n}$ with $x_{n} \in F\left(y_{n}\right), \forall n \in \mathbb{N}, x_{n} \rightarrow x$ weakly in $X$ implies $x \in F(y) . \quad F$ is called weakly completely continuous if $F$ has a weakly sequentially closed graph and, if $A$ is a bounded subset of $Z$, then $F(A)$ is a weakly relative compact subset of $X$.

Let now $(T, \mathcal{T}, \mu)$ be a measure space; a multifunction $F: T \rightarrow P_{f}(X)$ is said to be measurable (weakly measurable) if $F^{-1}(B) \in \mathcal{\sigma}$ for every closed (open) subset $B$ of $X$. If some values of $F$ are empty subsets of $X$, then $F$ is measurable if $T_{0}=$ $\{t \in T: F(t)=\emptyset\}$ belongs to $\sigma$ and on $T-T_{0} F$ is weakly measurable.

For a function $V$ defined in a Banach space $Y$, with values in a Banach space $X$ we recall the following definitions. $V$ is said to be bounded if $V(A)$ is bounded in $X$ for every bounded subset $A$ of $Y$. $V$ is said to be compact if it is continuous and $V(A)$ is relative compact in $X$ for every bounded subset $A$ of $Y$. Finally, $V$ is said to be completely continuous if for every sequence $\left\{y_{n}\right\}_{n} \subset Y$ with $y_{n} \rightarrow y$ weakly in $Y$, we have that $\left\{V\left(y_{n}\right)\right\}_{n}$ weakly converges to $V(y)$ in $X$.

Now we denote by $X^{*}$ the dual space of the Banach space $X$ and by $((\cdot, \cdot))$ the dual brackets between $X^{*}$ and $X$. A subset $S$ of $X \times X^{*}$ is said to be monotone if $\forall\left(x, x^{*}\right), \quad\left(y, y^{*}\right) \in S$ we have that $\left(\left(x^{*}-y^{*}, x-y\right)\right) \geq 0 . \quad S$ is called maximal monotone if it is not properly contained in any other monotone subset of $X \times X^{*}$. Let $A: D(A) \subseteq X \rightarrow 2^{X^{*}}$ be a multivalued operator, where $D(A)=\{x \in A: A(x) \neq \emptyset\}$ denotes the domain of $A$. $A$ is called a monotone (maximal monotone) operator if $G r A$ is a monotone (maximal monotone) subset of $X \times X^{*}$.

Remark 1: We observe that if $X^{*}$ is a uniformly convex space, then every maximal monotone operator $A: D(A) \subseteq X \rightarrow 2^{X^{*}}$ is demiclosed which means that for every sequence $\left\{x_{n}\right\}_{n} \subset D(A)$ with $x_{n} \rightarrow x$ in $D(A)$ and for every sequence $\left\{y_{n}\right\}_{n} \subset X^{*}$ with $y_{n} \rightarrow y$ weakly in $X^{*},\left(x_{n}, y_{n}\right) \in G r A, \forall n \in \mathbb{N}$ implies $(x, y) \in G r A$.

Let $A: D(A) \subseteq X \rightarrow 2^{X}$ be a set-valued operator with domain $D(A)$. We say that 
$A$ is accretive, if for every $x_{1}, x_{2} \in D(A)$, for every $y_{i} \in A\left(x_{i}\right), i=1,2$, and for every $\lambda>0$, we have $\left\|x_{1}-x_{2}\right\| \leq\left\|x_{1}-x_{2}+\lambda\left(y_{1}-y_{2}\right)\right\|$. Another equivalent definition can be given using the duality map of $X$, which is the set-valued function $J: X \rightarrow 2^{X^{*}}$ defined as $J(x)=\left\{x^{*} \in X:\left(\left(x^{*}, x\right)\right)=\|x\|^{2}=\left\|x^{*}\right\|^{2}\right\}$. Clearly the values of $J$ are nonempty, closed, convex and bounded subsets of $X^{*}$. Moreover, we recall that if $X^{*}$ is strictly convex or locally uniformly convex, the duality map $J$ is single-valued. So $A$ is accretive if for every $y_{i} \in A\left(x_{i}\right), i=1,2$, there exists $x^{*} \in J\left(x_{1}-x_{2}\right)$ such that $\left(\left(x^{*}, y_{1}-y_{2}\right)\right) \geq 0$.

Moreover $A$ is said to be $m$-accretive if it is accretive and for each $\lambda>0, I+\lambda A$ is surjective, where $I$ is the identity operator of $X$. Obviously if $X$ is a Hilbert space the notion of accretive ( $m$-accretive) operator coincides with that of monotone (maximal monotone) operator.

If $X$ is a Banach space and $T$ is the closed interval $[0, b]$, we denote by $W^{m, p}(T, X)$ the space of the functions $u \in L^{p}(T, X)$ which have distributional derivatives $u^{(k)}, k=1, \ldots, m$, which belong to the space $L^{p}(T, X)$. It is known (see $\left[3\right.$, p. 18]) that the Sobolev space $W^{m, p}(T, X)$ is a Banach space with the norm defined by

$$
\|u\|_{m, p}=\|u\|_{p}+\sum_{k=1}^{m}\left\|u^{(k)}\right\|_{p} .
$$

Moreover, if $X$ is reflexive then $W^{m, p}(T, X)$ can be identified with the space of absolutely continuous functions which have strong derivatives $u^{(k)}, k=1, \ldots, m$, with the property that $u^{(k)}, k=1, \ldots, m-1$, is absolutely continuous and $u^{(m)} \in L^{p}(T, X)$.

\section{Existence of Extremal Solutions}

Let $T-[0, b]$. We start with the following second order boundary value problem for differential inclusion:

$$
\left\{\begin{array}{c}
-x^{\prime \prime}(t) \in F\left(t, x(t), x^{\prime}(t)\right), \\
\left(B_{0} x\right)(0)=v_{0},\left(B_{1} x\right)(b)=v_{1},
\end{array}\right.
$$

where $F: T \times \mathbb{R} \times \mathbb{R} \rightarrow 2^{R}$ is a multifunction with nonempty, compact, convex values and $\quad v_{0}, v_{1} \in \mathbb{R}, \quad\left(B_{0} x\right)(0)=a_{0} x(0)-c_{0} x^{\prime}(0), \quad\left(B_{1} x\right)(b)=a_{1} x(b)+c_{1} x^{\prime}(b), \quad$ with $a_{0}, a_{1}, c_{0}, c_{1} \geq 0, a_{0}\left(a_{1} b+c_{1}\right)+c_{0} a_{1} \neq 0$. Note that if $c_{0}=c_{1}=v_{0}=v_{1}=0$ then we have the Dirichlet problem.

For problem (1), we give the definition of lower and upper solution: a function $\Psi \in W^{2,1}(T, \mathbb{R})$ is said to be a lower solution for problem (1) if

$$
\left\{\begin{array}{c}
-F\left(t, \Psi(t), \Psi^{\prime}(t)\right) \cap\left(-\infty, \Psi^{\prime \prime}(t)\right] \neq \emptyset \text { a.e. on } T \\
\left(B_{0} \Psi\right)(0) \leq v_{0}, \quad\left(B_{1} \Psi\right)(b) \leq v_{1} .
\end{array}\right.
$$

Since $F$ has nonempty, compact and convex values in $\mathbb{R}$, we can represent $F$ as

$$
F(t, x, y)=[\underline{f}(t, x, y), \bar{f}(t, x, y)], \quad \forall(t, x, y) \in T \times \mathbb{R} \times \mathbb{R},
$$

where $\bar{f}, \underline{f}: T \times \mathbb{R} \times \mathbb{R} \rightarrow \mathbb{R}$ are suitable functions.

So we can say that $\Psi \in W^{2,1}(T, \mathbb{R})$ is a lower solution of problem (1) if 


$$
\left\{\begin{array}{c}
-\Psi^{\prime \prime}(t) \leq \bar{f}\left(t, \Psi(t), \Psi^{\prime}(t)\right) \text { a.e. on } T \\
\left(B_{0} \Psi\right)(0) \leq v_{0}, \quad\left(B_{1} \Psi\right)(b) \leq v_{1} .
\end{array}\right.
$$

A function $\phi \in W^{2,1}(T, \mathbb{R})$ is said to be an upper solution for problem (1) if

$$
\left\{\begin{array}{c}
-F\left(t, \phi(t), \phi^{\prime}(t)\right) \cap\left[\phi^{\prime \prime}(t),+\infty\right) \neq \emptyset \text { a.e. on } T \\
\left(B_{0} \phi\right)(0) \geq v_{0}, \quad\left(B_{1} \phi\right)(b) \geq v_{1} .
\end{array}\right.
$$

So, using representation $(3.1)$ of $F$, we say that $\phi \in W^{2,1}(T, \mathbb{R})$ is an upper solution of problem (1) if

$$
\left\{\begin{array}{c}
-\phi^{\prime \prime}(t) \geq \underline{f}\left(t, \phi(t), \phi^{\prime}(t)\right) \text { a.e. on } T \\
\left(B_{0} \phi\right)(0) \geq v_{0}, \quad\left(B_{1} \phi\right)(b) \geq v_{1}
\end{array}\right.
$$

A function $x: T \rightarrow \mathbb{R}$ is a solution of problem (1) if $x \in W^{2,1}(T, \mathbb{R})$ and

$$
\left\{\begin{array}{c}
-x^{\prime \prime}(t) \in F\left(t, x(t), x^{\prime}(t)\right) \text { a.e. on } T \\
\left(B_{0} x\right)=v_{0},\left(B_{1} x\right)(b)=v_{1} .
\end{array}\right.
$$

Moreover, a solution $x_{*}$ of problem (1) is called minimal solution if for every solution $x$ of problem (1), we have that $x_{*}(t) \leq x(t), \forall t \in T$.

Analogously, a solution $x^{*}$ is said to be a maximal solution if for every solution $x$ of problem (1) we have that $x(t) \leq x^{*}(t), \forall t \in T$. The functions $x_{*}, x^{*}$ are called extremal solutions of problem (1).

Now we shall prove a sufficient condition for the existence of extremal solutions for problem (1). We shall need the following conditions:

$H(F)_{1}: \quad F: T \times \mathbb{R} \times \mathbb{R} \rightarrow P_{k c}(\mathbb{R})$ is a multifunction with the following properties:

(i) $\forall x, y \in \mathbb{R}, t \mapsto F(t, x, y)$ is measurable,

(ii) for a.e. $t \in T,(x, y) \mapsto F(t, x, y)$ is (u.s.c.),

(iii) $\forall r>0, \exists \gamma_{r} \in L^{1}\left(T, \mathbb{R}^{+}\right)$such that $\|F(t, x, y)\| \leq \gamma_{r}(t)$ for a.e. $t \in T$ and $\forall x, y \in \mathbb{R}$ with $|x|,|y| \leq r$.

$H_{0}$ : There exist a lower solution $\Psi$ and an upper solution $\phi$ of problem (1) with $\Psi(t) \leq \phi(t), \forall t \in T$, and there exists a function $h \in C\left(\mathbb{R}_{+},(0,+\infty)\right)$ such that $\|F(t, x, y)\| \leq h(|y|)$ for all $t \in T$ and all $x, y \in \mathbb{R}$ with $\Psi(t) \leq$ $x \leq \phi(t)$ and

$$
\int_{\lambda}^{\infty} \frac{r d r}{h(r)}>\max _{t \in T} \phi(t)-\min _{t \in T} \Psi(t)
$$

where $\lambda=\frac{1}{b} \max \{|\Psi(b)-\phi(0)|,|\Psi(b)-\phi(0)|\}$.

Remark 2: Condition $H_{0}$ is known as "Nagumo growth condition" and guarantees an a priori $L^{\infty}$-bound for the first derivative of every solution of problem (1). In fact, using a similar proof to that of Lemma 1.4.1 of [5], it is possible to prove that there exists $N_{1}>0$ (depending only on $\Psi, \phi$ and $h$ ) such that for all $x \in W^{2,1}(T, \mathbb{R}$ ) with $-x^{\prime \prime}(t) \in F\left(t, x(t), x^{\prime}(t)\right)$, a.e. $t \in T$ such that

$$
x \in[\Psi, \phi]=\left\{x \in W^{2,1}(T, \mathbb{R}): \Psi(t) \leq x(t) \leq \phi(t), \forall t \in T\right\},
$$


we have that $\left|x^{\prime}(t)\right| \leq N_{1}, \forall t \in T$.

In the following, $\forall a \in \mathbb{R}$ and for any subset $B \subset \mathbb{R}$ we will use the notations:

$$
\begin{aligned}
& B \vee a=\left\{\begin{array}{cc}
B \cap[a,+\infty) & \text { if } B \cap[a,+\infty) \neq \emptyset, \\
\{a\} & \text { otherwise },
\end{array}\right. \\
& B \wedge a=\left\{\begin{array}{cc}
B \cap(-\infty, a] & \text { if } B \cap(-\infty, a] \neq \emptyset, \\
\{a\} & \text { otherwise. }
\end{array}\right.
\end{aligned}
$$

We have the following existence result for problem (1).

Theorem 1: If hypotheses $H_{0}$ and $H(F)_{1}$ hold, then problem (1) has extremal solutions in the order interval $[\Psi, \phi]$.

Proof: Let $S_{1}$ be the set

$$
S_{1}=\{x \in[\Psi, \phi]: x \text { is a solution of }(1)\} .
$$

From Theorem 5.2 of $[20]$ we have that $S_{1} \neq \emptyset$. Consider, in the space $W^{2,1}(T, \mathbb{R})$, the order structure defined by

$$
x \prec y \Leftrightarrow x(t) \leq y(t), \forall t \in T .
$$

Then we shall prove that $S_{1}$ is an inductive and directed set with previous order structure. To this end, let $C$ be a chain in $S_{1}$. Since $L^{1}(T, \mathbb{R})$ is a complete lattice and $C$ is a bounded subset of $L^{1}(T, \mathbb{R})$, if $x=\sup C$, by Corollary IV.II.7 of [11], there exists a sequence $\left\{x_{n}\right\}_{n} \subset C$ such that $x=\sup \left\{x_{n}\right\}_{n}$ and $x \in L^{1}(T, \mathbb{R})$. By the monotone convergence theorem (cf. [6, Theorem IV.1]) we obtain that $x_{n} \rightarrow x$ in $L^{1}(T, \mathbb{R})$. Now, put $r=\max \left\{N_{1},\left\|\Psi^{\prime}\right\|_{\infty},\left\|\phi^{\prime}\right\|_{\infty}\right\}$ (cf. Remark 2). From condition $H(F)_{1}(i i i)$ we have that $\left|x_{n}^{\prime \prime}(t)\right| \leq \gamma_{r}(t)$, a.e. on $T, \forall n \in \mathbb{N}$. Hence $\left\{x_{n}\right\}_{n}$ is bounded in $W^{2,1}(T, \mathbb{R})$ and the set $\left\{x_{n}^{\prime \prime}\right\}_{n}$ is uniformly integrable.

Since the space $W^{2,1}(T, \mathbb{R})$ embeds compactly in $W^{1,1}(T, \mathbb{R})$ and continuously in $C^{1}(T, \mathbb{R})$ (cf. [1, pp. 100 and 144]), by the Dunford-Pettis Theorem applied to the sequence $\left\{x_{n}^{\prime \prime}\right\}_{n}$ (by passing to a subsequence if necessary) we deduce that $x \in C^{1}(T, \mathbb{R}), x_{n}(t) \rightarrow x(t), x_{n}^{\prime}(t) \rightarrow x^{\prime}(t), \forall t \in T$ and $x_{n}^{\prime \prime} \rightarrow y$ weakly in $L^{1}(T, \mathbb{R})$.

Taking into account that $\int_{0}^{t} x_{n}^{\prime \prime}(s) d s \rightarrow \int_{0}^{t} y(s) d s, \forall t \in T$ (cf. [43, p. 180]) and that $x_{n}^{\prime}(t)=x_{n}^{\prime}(0)+\int{ }_{0}^{t} x_{n}^{\prime \prime}(s) d s, \forall n \in \mathbb{N}, \forall t \in T$, we obtain that $x^{\prime}(t)=x^{\prime}(0)+\int{ }_{0}^{t} y(s) d s$, $\forall t \in T$, and so $x^{\prime \prime}(t)=y(t)$ a.e. on $T$. Therefore $x_{n}^{\prime \prime} \rightarrow x^{\prime \prime}$ weakly in $W^{2,1}(T, \mathbb{R})$.

Moreover, since $x_{n}^{\prime \prime} \rightarrow x^{\prime \prime}$ weakly in $L^{1}(T, \mathbb{R})$, there exists a sequence $\left\{v_{k}\right\}_{k}$, $v_{k}=\sum_{m=k}^{\infty} \lambda_{m}^{k} x_{m}^{\prime \prime}$ (where $\lambda_{m}^{k}=0$ except for a finite number of $m$ 's in which $\lambda_{m}^{k}>0$ and $\left.\sum_{m=k}^{\infty} \lambda_{m}^{k}=1\right)$ which converges to $x^{\prime \prime}$ in $L^{1}(T, \mathbb{R})$. By passing to a subsequence, if necessary, we may assume that $v_{k}(t) \rightarrow x_{k}^{\prime \prime}(t)$, a.e. on $T$.

We fix $t \in T$ such that $(x, y) \mapsto F(t, x, y)$ is (u.s.c.) in $\mathbb{R} \times \mathbb{R}, \quad-x_{n}^{\prime \prime}(t) \in$ $F\left(t, x_{n}(t), x_{n}^{\prime}(t)\right), \forall n \in \mathbb{N}$ and $v_{k}(t) \rightarrow x_{k}^{\prime \prime}(t)$. For every $\varepsilon>0$, since $F(t, \cdot, \cdot)$ is (u.s.c.) in the point $\left(x(t), x^{\prime}(t)\right)$, it is possible to find $\bar{n} \in \mathbb{N}$ such that

$$
\forall k \geq \bar{n} \Rightarrow-v_{k}(t)=-\sum_{m=k}^{\infty} \lambda_{m}^{k} x_{m}^{\prime \prime}(t) \in F\left(t, x(t), x^{\prime}(t)\right)+[-\varepsilon, \varepsilon],
$$

which implies that $-x^{\prime \prime}(t) \in F\left(t, x(t), x^{\prime}(t)\right)+[-\varepsilon, \varepsilon]$. Therefore, since $\varepsilon$ is arbitrary, it follows that $x \in S_{1}$. Using Zorn's Lemma, we infer that $S_{1}$ has a maxi- 
mal element $x^{*} \in S_{1}$. In a similar way we prove that in $S_{1}$ there is an element $x_{*}$ which is minimal.

Finally, we show that $S_{1}$ is directed (i.e., if $x_{1}, x_{2} \in S_{1}$ then there exists $x \in S_{1}$ such that $x_{1} \prec x$ and $\left.x_{2} \prec x\right)$. To this end, let $x_{1}, x_{2} \in S_{1}$ and let $x_{3}=\max \left\{x_{1}, x_{2}\right\}$. Since $x_{1}, x_{2} \in W^{2,1}(T, \mathbb{R})$ and $x_{3}=\left(x_{1}, x_{2}\right)_{+}+x_{2}$, from Lemma 7.6 of [27], it follows that $x_{3} \in W^{1,1}(T, \mathbb{R})$ and

$$
x_{3}^{\prime}(t)= \begin{cases}x_{1}^{\prime}(t) & \text { if } x_{1}(t) \geq x_{2}(t), \\ x_{2}^{\prime}(t) & \text { if } x_{1}(t) \leq x_{2}(t),\end{cases}
$$

a.e. on $T$. First suppose that $x_{3} \in W^{2,1}(T, \mathbb{R})$. Since $x_{3} \in C^{1}(T, \mathbb{R})$, we have that at the points $t \in T$ at which $x_{1}$ and $x_{2}$ coincide, $x_{1}^{\prime}(t)$ is equal to $x_{2}^{\prime}(t)$ and so

$$
x_{3}^{\prime}(t)= \begin{cases}x_{1}^{\prime}(t) & \text { if } x_{1}(t)>x_{2}(t), \\ x_{2}^{\prime}(t) & \text { if } x_{1}(t)<x_{2}(t), \\ x_{1}^{\prime}(t) & \text { if } x_{1}(t)=x_{2}(t),\end{cases}
$$

for every $t \in T$. Let

and

$$
T_{0}=\left\{t \in T: x_{1}^{\prime}(t)=x_{2}^{\prime}(t) \text { and } x_{1}^{\prime \prime}(t) \neq x_{2}^{\prime \prime}(t)\right\}
$$

$$
T^{\prime}=\left\{t \in T: \exists x_{1}^{\prime \prime}(t) \text { and } \exists x_{2}^{\prime \prime}(t)\right\} .
$$

From the Banach Lemma (cf. [20, Lemma A.9]) we obtain that $m T_{0}=0$. Moreover, for every $t \in T^{\prime} \backslash T_{0}$ we have that if $x_{1}(t)>x_{2}(t)$, then $x_{3}^{\prime \prime}(t)=x_{1}^{\prime \prime}(t)$; while if $x_{1}(t)<x_{2}(t)$ then $x_{3}^{\prime \prime}(t)=x_{2}^{\prime \prime}(t)$. Finally, if $x_{1}(t)=x_{2}(t)$ then $x_{3}^{\prime \prime}(t)=x_{1}^{\prime \prime}(t)=x_{2}^{\prime \prime}(t)$. Therefore, it follows that

$$
x_{3}^{\prime \prime}(t)= \begin{cases}x_{1}^{\prime \prime}(t) & \text { if } x_{1}(t) \geq x_{2}(t), \\ x_{2}^{\prime \prime}(t) & \text { if } x_{1}(t) \leq x_{2}(t),\end{cases}
$$

$\forall t \in T^{\prime} \backslash T_{0}$. From this we deduce that $-x_{3}^{\prime \prime}(t) \in F\left(t, x_{3}(t), x_{3}^{\prime}(t)\right)$ a.e. on $T$, which means that $x_{3} \in S_{1}$. So $x_{3}$ is the element in $S_{1}$ such that $x_{1} \prec x_{3}$ and $x_{2} \prec x_{3}$.

In the case in which $x_{3} \notin W^{2,1}(T, \mathbb{R})$ we consider the following truncation operator $\tau_{3}: W^{1,1}(T, \mathbb{R}) \rightarrow W^{1,1}(T, \mathbb{R})$ defined by

$$
\tau_{3}(x)(t)=\left\{\begin{array}{cc}
\phi(t) & \text { if } x(t) \geq \phi(t), \\
x(t) & \text { if } x_{3}(t) \leq x(t) \leq \phi(t), \\
x_{3}(t) & \text { if } x(t) \leq x_{3}(t),
\end{array}\right.
$$

for every $x \in W^{1,1}(T, \mathbb{R})$ and for all $t \in T$. By Lemma 7.6 of [27], we get that

$$
\tau_{3}(x)^{\prime}(t)=\left\{\begin{array}{cc}
\phi^{\prime}(t) & \text { if } x(t) \geq \phi(t), \\
x^{\prime}(t) & \text { if } x_{3}(t) \leq x(t) \leq \phi(t), \\
x_{3}^{\prime}(t) & \text { if } x(t) \leq x_{3}(t),
\end{array}\right.
$$

$\forall x \in W^{1,1}(T, \mathbb{R})$ and for a.e. $t \in T$. Put $N=1+\max \left\{N_{1},\left\|\Psi^{\prime}\right\|_{\infty},\left\|\phi^{\prime}\right\|_{\infty}\right\}$ (cf. 
Remark 2) and denote by $q_{N}$ and $u_{3}$ the truncation function and the penalty function respectively, which are defined by $q_{N}: \mathbb{R} \rightarrow \mathbb{R}$ with

$$
q_{N}(x)=\left\{\begin{array}{cc}
N & \text { if } x \geq N, \\
x & \text { if }-N \leq x \leq N, \\
-N & \text { if } x \leq-N,
\end{array}\right.
$$

$\forall x \in \mathbb{R}$, and $u_{3}: T \times \mathbb{R} \rightarrow \mathbb{R}$ where

$$
u_{3}(t, x)=\left\{\begin{array}{cc}
x-\phi(t) & \text { if } x \geq \phi(t), \\
0 & \text { if } x_{3}(t) \leq x \leq \phi(t), \\
x-x_{3}(t) & \text { if } x \leq x_{3}(t),
\end{array}\right.
$$

$\forall(t, x) \in T \times \mathbb{R}$. Now let $\tilde{F}: T \times \mathbb{R} \times \mathbb{R} \rightarrow 2^{\mathbb{R}} \backslash\{\emptyset\}$ be the multifunction defined by

$$
\tilde{F}(t, x, y)=\overline{c o}\left(\bigcup_{i=1}^{3} F_{i}(t, x, y)\right), \quad \forall(t, x, y) \in T \times \mathbb{R} \times \mathbb{R},
$$

where $F_{i}: T \times \mathbb{R} \times \mathbb{R} \rightarrow 2^{\mathbb{R}}, \quad i=1,2,3$, are the multifunctions defined by

$$
\begin{gathered}
F_{1}(t, x, y)=\left\{\begin{array}{cc}
F(t, x, y) & \text { if } x_{3}(t) \leq x \leq \phi(t), \\
\emptyset & \text { otherwise, }
\end{array}\right. \\
F_{2}(t, x, y)=\left\{\begin{array}{cc}
F\left(t, x_{3}(t), y\right) \vee\{\tilde{x}(t)\} & \text { if } x_{3}(t) \geq x, \\
\emptyset & \text { otherwise }
\end{array}\right. \\
F_{3}(t, x, y)=\left\{\begin{array}{cc}
F(t, \phi(t), y) \wedge\left\{-\phi^{\prime \prime}(t)\right\} & \text { if } \phi(t) \leq x, \\
\emptyset & \text { otherwise }
\end{array}\right.
\end{gathered}
$$

$\forall(t, x, y) \in T \times \mathbb{R} \times \mathbb{R}$, and $\widetilde{x} \in L^{1}(T, \mathbb{R})$ is the function

$$
\tilde{x}(t)=\max \left\{-x_{1}^{\prime \prime}(t),-x_{2}^{\prime \prime}(t)\right\}, \text { a.e. on } T \text {. }
$$

It is obvious that $\tilde{F}$ has nonempty, closed and convex values. We shall prove that, for every $(x, y) \in \mathbb{R} \times \mathbb{R}$, the multifunction $t \mapsto \widetilde{F}(t, x, y)$ is measurable. To this end, it suffices to show that $\forall(x, y) \in \mathbb{R} \times \mathbb{R}, t \mapsto F_{i}(t, x, y)$ is weakly measurable (cf. [32, Proposition 2.3 and Theorem 9.1]). If $i=1$, then the measurability of $t \mapsto F_{1}(t, x, y)$ follows by observing that, for every open subset $A$ of $\mathbb{R}$, the sets $T_{0}=\left\{t \in T: F_{1}(t, x, y)=\emptyset\right\}$ and $F_{1}^{-1}(A)$ are measurable. If $i=2$, first we observe that

$$
F_{2}(t, x, y)=\left\{\begin{array}{cc}
\emptyset & \text { if } x(t)>x_{3}(t), \\
F\left(t, x_{3}(t), y\right) \cap[\tilde{x}(t),+\infty) & \text { if } \tilde{x}(t)<\bar{f}\left(t, x_{3}(t), y\right) \text { and } \\
\{\tilde{x}(t)\} & x(t) \leq x_{3}(t), \\
& \text { if } \tilde{x}(t) \geq \bar{f}\left(t, x_{3}(t), y\right) \text { and } \\
& x(t) \leq x_{3}(t),
\end{array}\right.
$$


$\forall(t, x, y) \in T \times \mathbb{R} \times \mathbb{R}$. Moreover, since the set $\left\{t \in T: F_{2}(t, x, y)=\emptyset\right\}$ is measurable and also since the multifunctions $t \mapsto[\widetilde{x}(t),+\infty)$ and $t \mapsto F\left(t, x_{3}(t), y\right) \cap[\widetilde{x}(t),+\infty)$ are measurable (cf. [32, Theorem 4.1 and Theorem 6.4]) it follows $t \mapsto F_{2}(t, x, y)$ is measurable. In a similar way we obtain the measurability of the multifunction $t \mapsto F_{3}(t, x, y)$.

Now we shall show that, for a.e. $t \in T$, the multifunction $(x, y) \mapsto \widetilde{F}(t, x, y)$ is (u.s.c.) in $\mathbb{R} \times \mathbb{R}$. As for the measurability, using Theorem $3^{\prime}$ of [4], it is sufficient to prove that the maps $(x, y) \mapsto F_{i}(t, x, y), i=1,2,3$ are (u.s.c.) in every $\left(x_{0}, y_{0}\right) \in \mathbb{R} \times \mathbb{R}$. Let $i=1$. If $x_{0}<x_{3}(t)$ or $x_{0}>\phi(t)$ it is possible to find a neighborhood $U$ of $\left(x_{0}, y_{0}\right)$ such that $F_{1}(t, x, y)=\emptyset, \forall(x, y) \in U$, and so $(x, y) \mapsto F_{1}(t, x, y)$ is (u.s.c.) in $\left(x_{0}, y_{0}\right)$. Instead, if $x_{3}(t) \leq x_{0} \leq \phi(t)$, then the upper semicontinuity of $(x, y) \mapsto F_{1}(t, x, y)$ in $\left(x_{0}, y_{0}\right)$ follows directly from $H(F)_{1}(i i)$. If $i=2$, observe that

$$
F_{2}(t, x, y)=\left\{\begin{array}{cc}
\emptyset & \text { if } x>x_{3}(t), \\
F\left(t, x_{3}(t), y\right) & \text { if } x \leq x_{3}(t) \text { and } \tilde{x}(t) \leq \underline{f}\left(t, x_{3}(t), y\right), \\
{\left[\tilde{x}(t), \bar{f}\left(t, x_{3}(t), y\right)\right]} & \text { if } x \leq x_{3}(t) \text { and } \\
\{\tilde{x}(t)\} & \underline{f}\left(t, x_{3}(t), y\right)<\tilde{x}(t)<\bar{f}\left(t, x_{3}(t), y\right), \\
& \text { if } x \leq x_{3}(t) \text { and } \tilde{x}(t) \geq \bar{f}\left(t, x_{3}(t), y\right),
\end{array}\right.
$$

$\forall(t, x, y) \in T \times \mathbb{R} \times \mathbb{R}$. Now fix an open subset $A$ of $\mathbb{R}$ such that $A \supset F_{2}\left(t, x_{0}, y_{0}\right)$. If $x_{0}>x_{3}(t)$, then we can find a neighborhood $U$ of $\left(x_{0}, y_{0}\right)$ such that $F_{2}(t, x, y)=\emptyset$, $\forall(x, y) \in U$. If $x_{0} \leq x_{3}(t)$ and $\tilde{x}(t) \leq f\left(t, x_{3}(t), y_{0}\right)$, choose $\varepsilon>0$ such that $\left[\underline{f}\left(t, x_{3}(t), y_{0}\right)-\varepsilon, \bar{f}\left(t, x_{3}(t), y_{0}\right)+\varepsilon\right] \subset A$. Since the functions $y \mapsto f\left(t, x_{3}(t), y\right)$ and $y \mapsto \bar{f}\left(t, x_{3}(t), y\right)$ are (l.s.c.) and (u.s.c.) respectively in $y_{0}$, there exists a neighborhood $U$ of $\left(x_{0}, y_{0}\right)$ with the property

$$
A \supset\left[\underline{f}\left(t, x_{3}(t), y\right), \bar{f}\left(t, x_{3}(t), y\right)\right] \supset F_{2}(t, x, y), \forall(x, y) \in U .
$$

In the case in which $x_{0} \leq x_{3}(t)$ and $\underline{f}\left(t, x_{3}(t), y_{0}\right)<\tilde{x}(t)<\bar{f}\left(t, x_{3}(t), y_{0}\right)$ choose $\varepsilon>0$ such that $\left[\tilde{x}(t), \bar{f}\left(t, x_{3}(t), y\right)+\varepsilon\right] \bar{\subset} A$. So from the upper semicontinuity of $y \mapsto \bar{f}\left(t, x_{3}(t), y\right)$ we can find a neighborhood $U$ of $\left(x_{0}, y_{0}\right)$ such that

$$
A \supset F_{2}(t, x, y), \quad \forall(x, y) \in U \text {. }
$$

Next, we consider the case in which $x_{0} \leq x_{3}(t)$ and $\tilde{x}(t) \geq \bar{f}\left(t, x_{3}(t), y_{0}\right)$. Using again the upper semicontinuity of $y \mapsto \bar{f}\left(t, x_{3}(t), y\right)$ at $y_{0}$ and the fact that $\widetilde{x}(t) \in A$, as above, we deduce the existence of a neighborhood $U$ of $\left(x_{0}, y_{0}\right)$ such that $A \supset$ $F_{2}(t, x, y), \forall(x, y) \in U$. Similarly, we obtain the upper semicontinuity of $(x, y) \mapsto$ $F_{3}(t, x, y)$.

Finally we shall prove that $\widetilde{F}$ is integrably bounded on the bounded subsets of $L^{1}(T, \mathbb{R})$. To this end, fix $r>0$ and $x, y \in \mathbb{R}$ such that $|x| \leq r$ and $|y| \leq r$. From $H(F)_{1}(i i i)$ it follows that there exists a function $\tilde{\gamma}_{r} \in L^{1}\left(T, \mathbb{R}_{0}^{+}\right)$such that $\|F(t, x, y)\| \leq \tilde{\gamma}_{r}(t)$ a.e. on $T$. Now let $R=\max \left\{\|\Psi\|_{\infty},\|\phi\|_{\infty}, r\right\}$ and let $\gamma_{R} \in L^{1}\left(T, \mathbb{R}_{0}^{+}\right)$be the function corresponding to $R$ from condition $H(F)_{1}(i i i)$. Putting $\gamma_{r}=\tilde{\gamma}_{r}+\gamma_{R}+\left|\phi^{\prime \prime}\right|+|\widetilde{x}|$, it is evident that $\gamma_{r} \in L^{1}\left(T, \mathbb{R}_{0}^{+}\right)$. So, if $x_{3}(t)<x<\phi(t)$, then $\widetilde{F}(t, x, y)=F(t, x, y)$ and so $|\widetilde{F}(t, x, y)| \leq \gamma_{r}(t)$, a.e. on $T$. Instead, if $x_{3}(t)>x$ (or analogously $x>\phi(t)$ ) we have that 


$$
\tilde{F}(t, x, y)=\left\{\begin{array}{cc}
F\left(t, x_{3}(t), y\right) & \text { if } \tilde{x}(t) \leq \underline{f}\left(t, x_{3}(t), y\right), \\
{\left[\tilde{x}(t), \bar{f}\left(t, x_{3}(t), y\right)\right]} & \text { if } \underline{f}\left(t, x_{3}(t), y\right)<\tilde{x}(t)<\bar{f}\left(t, x_{3}(t), y\right), \\
\{\tilde{x}(t)\} & \text { if } \tilde{x}(t) \geq \bar{f}(t, \Psi(t), y)
\end{array}\right.
$$

and so, in any case, it follows that $|\tilde{F}(t, x, y)| \leq \gamma_{r}(t)$ a.e. on $T$. Finally, if $x=$ $x_{3}(t)$ (or analogously $x=\phi(t)$ ), we have that

$$
\tilde{F}(t, x, y)=\left\{\begin{array}{cc}
F\left(t, x_{3}(t), y\right) & \text { if } \tilde{x}(t) \leq \bar{f}\left(t, x_{3}(t), y\right), \\
{\left[\underline{f}\left(t, x_{3}(t), y\right), \tilde{x}(t)\right]} & \text { if } \tilde{x}(t)>\bar{f}\left(t, x_{3}(t), y\right),
\end{array}\right.
$$

which gives the expected equality $|\tilde{F}(t, x, y)| \leq \gamma_{r}(t)$ a.e. on $T$.

Consider now the following boundary value problem:

$$
\left\{\begin{array}{c}
-x^{\prime \prime}(t) \in \tilde{F}\left(t, x(t), q_{N}\left(\tau_{3}(x)^{\prime}(t)\right)\right)-u_{3}(t, x(t)) \text { a.e. on } T \\
\left(B_{0} x\right)(0)=\nu_{0},\left(B_{1} x\right)(b)=\nu_{1}
\end{array}\right.
$$

We shall prove that $(2)$ has a solution in $W^{2,1}(T, \mathbb{R})$. Let $H: W^{1,1}(T, \mathbb{R}) \rightarrow 2^{L^{1}(T, \mathbb{R})}$ be defined by $H(x)=\left\{z \in L^{1}(T, \mathbb{R}): z(t) \in \widetilde{F}\left(t, x(t), q_{N}\left(\tau_{3}(x)^{\prime}(t)\right)\right)-u_{3}(t, x(t))+x(t)\right.$ a.e. on $T\}, \forall x \in W^{1,1}(T, \mathbb{R})$. From the properties of the multifunction $\widetilde{F}$ and from the continuity of the function $t \mapsto q_{N}\left(\tau_{3}(x)^{\prime}(t)\right)$, we deduce that $H(x) \neq \emptyset$, $\forall x \in W^{1,1}(T, \mathbb{R})$ and, denoting $r=\max \left\{N,\|\Psi\|_{\infty},\|\phi\|_{\infty}\right\}$ by $H(F)_{1}(i i i)$ it follows that

$$
\|H(x)\|_{1} \leq\left\|\gamma_{r}\right\|_{1}+r b
$$

Moreover, let $\widehat{L}: D \subseteq L^{1}(T) \rightarrow L^{1}(T)$ be defined by $\widehat{L} x=-x^{\prime \prime}$, for every $x \in D$, where $D=\left\{x \in W^{2,1}(T, \mathbb{R}):\left(B_{0} x\right)(0)=v_{0},\left(B_{1} x\right)(b)=v_{1}\right\}$. From [38] we have that $\widehat{L}$ is $m$ accretive and so (cf. $[3$, p. 72$])$ the operator $L^{-1}=(I+\widehat{L})^{-1}: L^{1}(T, \mathbb{R}) \rightarrow D \subset$ $L^{1}(T, \mathbb{R})$ is well-defined, linear and compact (cf. [38]).

Now let $\Gamma: W^{1,1}(T, \mathbb{R}) \rightarrow 2^{W^{1,1}}(T, \mathbb{R})$ be the multivalued operator defined by $\Gamma(x)=L^{-1} H(x), \forall x \in W^{1,1}(T, \mathbb{R})$. From the properties of $L^{-1}$ and from the condition $H(F)_{1}$, it is easy to show that $\Gamma$ has nonempty, convex and compact values and by (3.2) we deduce that $\Gamma$ is a compact operator. Now we shall prove that $\Gamma$ has a closed graph. To this end, from the continuity of the operator $L^{-1}$, it is sufficient (cf. [40, Proposition 1.5]) to prove that $H$ is weakly completely continuous. Denote by $G: W^{1,1}(T, \mathbb{R}) \rightarrow 2^{L^{1}(T, \mathbb{R})}$ the Nemtyskii operator

$$
G(x)=\left\{z \in L^{1}(T, \mathbb{R}): z(t) \in \widetilde{F}\left(t, x(t), q_{N}\left(\tau_{3}(x)^{\prime}(t)\right)\right) \text { a.e. on } T\right\},
$$

$\forall x \in W^{1,1}(T, \mathbb{R})$. We shall prove that $G$ has a weakly sequentially closed graph. To this end, let $\left\{x_{n}\right\}_{n}$ be a sequence which converges to $x$ in $W^{1,1}(T, \mathbb{R})$ and $\left\{z_{n}\right\}_{n}$ be a sequence in $L^{1}(T, \mathbb{R})$ such that $z_{n} \rightarrow z$ weakly in $L^{1}(T, \mathbb{R})$ for $z_{n} \in G\left(x_{n}\right), \forall n \in \mathbb{N}$. Applying Mazur's Theorem to the sequence $\left\{z_{n}\right\}_{n}$, we obtain that there exists a sequence $\left\{v_{n}\right\}_{n}, v_{n} \in \operatorname{co}\left\{z_{m}: m \geq n\right\}$, such that $v_{n} \rightarrow z$ in $L^{1}(T, \mathbb{R})$. Passing to a subsequence if necessary, we may suppose that 


$$
v_{n}(t) \rightarrow z(t) \text { a.e. on } T \text {. }
$$

From the upper semicontinuity of $(x, y) \mapsto \tilde{F}(t, x, y)$ and since $q_{N}\left(\tau_{3}\left(x_{n}\right)^{\prime}(t)\right) \rightarrow$ $q_{N}\left(\tau_{3}(x)^{\prime}(t)\right)$ a.e. on $T$, we deduce that for a.e. $t \in T$ and for every $\varepsilon>0$, there exists $n_{\varepsilon} \in \mathbb{N}$ such that

$$
\tilde{F}\left(t, x_{n}(t), q_{N}\left(\tau_{3}\left(x_{n}\right)^{\prime}(t)\right)\right) \subset \tilde{F}\left(t, x(t), q_{N}\left(\tau_{3}(x)^{\prime}(t)\right)+[-\varepsilon, \varepsilon], \forall n>n_{\varepsilon} .\right.
$$

Since $z_{n} \in G\left(x_{n}\right)$ and $\widetilde{F}$ has convex values, it follows that

$$
v_{n}(t) \in \tilde{F}\left(t, x(t), q_{N}\left(\tau_{3}(x)^{\prime}(t)\right)\right)+[-\varepsilon, \varepsilon], \quad \forall n>n_{\varepsilon}
$$

and so from (3.3) and recalling that $\widetilde{F}$ has closed values we conclude that $z \in G(x)$.

Moreover, taking into account that the function $x \mapsto x(\cdot)-u_{3}(\cdot, x(\cdot))$ is continuous from $W^{1,1}(T, \mathbb{R})$ into $L^{1}(T, \mathbb{R})$ (it is simple to deduce this by applying the dominated convergence theorem), we obtain that $H$ has a weakly sequentially closed graph and since $H$ maps bounded subsets of $W^{1,1}(T, \mathbb{R})$ into weakly relative compact subsets of $L^{1}(T, \mathbb{R})$, we get the weakly completely continuity of $H$.

Finally, we apply the Kakutani-Ky-Fan Theorem (cf. [20, Theorem A.2]) to $\Gamma$ and obtain that there exists $x \in D$ such that $x \in \Gamma(x)$. Therefore, $x$ is a solution of the problem (2).

Now, we shall show that every solution $x$ of problem (2) belongs to the order interval $\left[x_{3}, \phi\right]$. First observe that the boundary conditions on $x, x_{1}$ and $\phi$ imply that

$$
\begin{gathered}
\left(x_{1}^{\prime}-x^{\prime}\right)(0)\left(x_{1}-x\right)_{+}(0) \geq 0, \quad\left(x^{\prime}-\phi^{\prime}\right)(0)(x-\phi)_{+}(0) \leq 0, \\
\left(x_{1}^{\prime}-x^{\prime}\right)(b)\left(x_{1}-x\right)_{+}(b) \leq 0, \quad\left(x^{\prime}-\phi^{\prime}\right)(b)(x-\phi)_{+}(b) \geq 0 .
\end{gathered}
$$

In fact, we know that

$$
a_{0} x_{1}(0)-c_{0} x_{1}^{\prime}(0)=\nu_{0}=a_{0} x(0)-c_{0} x^{\prime}(0) \Rightarrow-c_{0}\left(x_{1}^{\prime}-x^{\prime}\right)(0)=a_{0}\left(x-x_{1}\right)(0) .
$$

If $c_{0}=0$, then $a_{0}>0$ and so $\left(x_{1}-x\right)_{+}(0)=0$. Therefore $\left(x_{1}^{\prime}-x^{\prime}\right)(0)\left(x_{1}-x\right)+(0)=$ 0 . If $c_{0}>0$, then

$$
-\left(x_{1}^{\prime}-x^{\prime}\right)(0)=\frac{a_{0}}{c_{0}}\left(x-x_{1}\right)(0)
$$

Now, if $x(0) \geq x_{1}(0)$ then $\left(x_{1}-x\right)_{+}(0)=0$ and so $\left(x_{1}^{\prime}-x^{\prime}\right)(0)\left(x_{1}-x\right)+(0)=0$, while if $x(0)<x_{1}(0)$ by $(3.5)$ we have that $\left(x_{1}^{\prime}-x^{\prime}\right)(0)>0$ and hence $\left(x_{1}^{\prime}-\right.$ $\left.x^{\prime}\right)(0)\left(x_{1}-x\right)+(0) \geq 0$. In an analogous way we obtain the other inequalities.

If $x \notin\left[x_{1}, \phi\right]$ then there exists $\bar{t}>0$ such that either $x(\bar{t})<x_{1}(\bar{t})$ or $x(\bar{t})>\phi(\bar{t})$. If $x(\bar{t})<x_{1}(\bar{t})$ (analogously we can proceed if $x(\bar{t})>\phi(\bar{t})$ ) it is possible to find an interval $\left[t_{1}, t_{2}\right] \subset T$ on which

$$
\begin{gathered}
x(t)<x_{1}(t) \quad \forall t \in\left(t_{1}, t_{2}\right), \\
x\left(t_{1}\right)=x_{1}\left(t_{1}\right) \text { or } t_{1}=0
\end{gathered}
$$

and

$$
x\left(t_{2}\right)=x_{1}\left(t_{2}\right) \text { or } t_{2}=b .
$$


In any case, at $t_{1}$ and $t_{2}$, we have (cf. (3.4))

$$
\left(x_{1}^{\prime}-x^{\prime}\right)\left(t_{1}\right)\left(x_{1}-x\right)\left(t_{1}\right) \geq 0, \quad\left(x_{1}^{\prime}-x^{\prime}\right)\left(t_{2}\right)\left(x_{1}-x\right)\left(t_{2}\right) \leq 0
$$

while $\forall t \in\left(t_{1}, t_{2}\right)$ it follows that $\tau_{3}(x)^{\prime}(t)=x_{3}^{\prime}(t)$ and $u_{3}(t, x(t))=\left(x-x_{3}\right)(t)$. Since $x$ is a solution of problem (2), we deduce that $-x^{\prime \prime}(t)+x(t)-x_{3}(t) \epsilon$ $F_{2}\left(t, x(t), x_{3}^{\prime}(t)\right)$ a.e. on $\left[t_{1}, t_{2}\right]$ and so $-x^{\prime \prime}(t)+x(t)-x_{3}(t) \geq \widetilde{x}(t) \geq-x_{1}^{\prime \prime}(t)$ a.e. on $\left[t_{1}, t_{2}\right]$.

Multiplying by $\left(x_{1}-x\right)(t)$ and integrating over $\left[t_{1}, t_{2}\right]$, we have

$$
\int_{t_{1}}^{t_{2}}\left(x_{1}^{\prime \prime}-x^{\prime \prime}\right)(t)\left(x_{1}-x\right)(t) d t \geq \int_{t_{1}}^{t_{2}}\left(x_{1}-x\right)^{2}(t) d t .
$$

From the integration by parts formula and from (3.6), we obtain

$$
\int_{t_{1}}^{t_{2}}\left(x_{1}^{\prime \prime}-x^{\prime \prime}\right)(t)\left(x_{1}-x\right)(t) d t \leq 0
$$

and so, since $\left(x_{1}-x\right)(t)>0, \forall t \in\left(t_{1}, t_{2}\right)$, from (3.7) we get a contradiction. Therefore we must have $x \in\left[x_{1}, \phi\right]$. Similarly, we can prove that $x \in\left[x_{2}, \phi\right]$ and so $x \in\left[x_{3}, \phi\right]$.

From this, we obtain that $x$ really is a solution for problem (1) and moreover $x_{1} \prec x$ and $x_{2} \prec x$, which was to be proved.

The proof of the previous theorem can be adapted to prove a similar result for the following periodic problem

$$
\left\{\begin{array}{c}
-x^{\prime \prime}(t) \in F\left(t, x(t), x^{\prime}(t)\right) \text { a.e. on } T \\
x(0)=x(b), x^{\prime}(0)=x^{\prime}(b)
\end{array}\right.
$$

where $F: T \times \mathbb{R} \times \mathbb{R} \rightarrow P_{k, c}(\mathbb{R})$ is a multifunction.

Obviously we say that a function $\Psi \in W^{2,1}(T, \mathbb{R})$ is a lower solution for problem (3) if

$$
\left\{\begin{array}{c}
-F\left(t, \Psi(t), \Psi^{\prime}(t)\right) \cap\left(-\infty, \Psi^{\prime \prime}(t)\right] \neq \emptyset \text { a.e. on } T \\
\Psi(0)=\Psi(b), \Psi^{\prime}(0) \geq \Psi^{\prime}(b)
\end{array}\right.
$$

and $\phi \in W^{2,1}(T, \mathbb{R})$ is an upper solution for problem (3) if

$$
\left\{\begin{array}{c}
-F\left(t, \phi(t), \phi^{\prime}(t)\right) \cap\left[\phi^{\prime \prime}(t)+\infty\right) \neq \emptyset \text { a.e. on } T \\
\phi(0)=\phi(b), \phi^{\prime}(0) \leq \phi^{\prime}(b)
\end{array}\right.
$$

Therefore we have the following result.

Theorem 2: If hypotheses $H_{0}$ and $H(F)_{1}$ hold, then problem (3) has extremal solutions in the order interval $[\Psi, \phi]$. 


\section{Boundary Value Problem in Hilbert Space}

Let $(H,\langle\cdot, \cdot\rangle)$ be a real separable Hilbert space. In this section we prove the existence of solutions for the following boundary value problem for differential inclusions:

$$
\left\{\begin{array}{c}
-x^{\prime \prime}(t) \in F\left(t, x(t), x^{\prime}(t)\right), \text { a.e. on } T \\
x(0)=v_{0}, x(b)=v_{1},
\end{array}\right.
$$

under the condition that $F: T \times H \times H \rightarrow P_{w k k}(H)$ is a multifunction satisfying the following hypotheses:

$H(F)_{2}: \quad F: T \times H \times H \rightarrow P_{w k k}(H)$ is a multifunction with the properties:

(i) $\forall x, y \in H, t \mapsto F(t, x, y)$ is measurable;

(ii) for a.e. $t$ in $T,(x, y) \mapsto F(t, x, y)$ is (u.s.c.);

(iii) $\forall r>0, \exists \gamma_{r} \in L^{2}\left(T, \mathbb{R}^{+}\right)$such that $\|F(t, x, y)\| \leq \gamma_{r}(t)$ for a.e.

and $v_{0}, v_{1} \in H$. $t \in T$ and $\forall x, y \in H$ with $\|x\| \leq r$;

A function $x: T \rightarrow H$ is a solution of problem (4) if $x \in W^{2,2}(T, H)$ and

$$
\left\{\begin{array}{c}
-x^{\prime \prime}(t) \in F\left(t, x(t), x^{\prime}(t)\right) \text { a.e. on } T \\
x(0)=v_{0}, x(b)=v_{1} .
\end{array}\right.
$$

Put $D=\left\{x \in W^{2,2}(T, H): x(0)=v_{0}, x(b)=v_{1}\right\}$, and let $\widehat{L}: D \subseteq L^{2}(T, H) \rightarrow L^{2}(T, H)$ be the operator defined by $\widehat{L}(x)=-x^{\prime \prime}, \forall x \in D$. First we prove the following properties on the operator $\widehat{L}$.

Proposition 3: $\widehat{L}$ is a maximal monotone operator.

Proof: From the integration by parts formula, it follows immediately that $\widehat{L}$ is monotone. So we need to show that $R(I+\widehat{L})=L^{2}(T, H)$ (cf. [3, Theorem 1.2]) or equivalently, that $\forall h \in L^{2}(T, H)$, the following Dirichlet boundary value problem

$$
\left\{\begin{array}{c}
-x^{\prime \prime}(t)+x(t)=h(t) \text { a.e. on } T \\
x(0)=v_{0}, x(b)=v_{b}
\end{array}\right.
$$

has a solution in $W^{2,2}(T, H)$.

To this end, we denote by $K$ the following closed and convex subset of $W^{1,2}(T, H)$

$$
K=\left\{x \in W^{1,2}(T, H): x(0)=v_{0}, x(b)=v_{1}\right\}
$$

and we consider the bilinear form $a: W^{1,2}(T, H) \times W^{1,2}(T, H) \rightarrow \mathbb{R}$ defined by

$$
a(u, v)=\int_{T} u^{\prime}(t) v^{\prime}(t) d t+\int_{T} u(t) v(t) d t .
$$

First note that $a$ is continuous, since $|a(u, v)|=\left|\langle u, v\rangle_{W^{1,2}}\right| \leq$ $\|u\|_{W^{1,2}}\|v\|_{W^{1,2}}$ and it is coercive since $a(v, v)=\|v\|_{W^{1,2}}^{2}$. By the Stampacchia theorem (cf. [6, Theorem V.6]), there exists a unique element $u \in K$ 
such that

$$
\int_{T} u^{\prime}(t)\left(v^{\prime}-u^{\prime}\right)(t) d t+\int_{T} u(t)(v-u)(t) d t \geq \int_{T} h(t)(v-u)(t) d t, \forall v \in K
$$

Putting $v=u+w$ in (4.1) with $w \in C_{c}^{1}(] 0, b[)$, we have that

$$
\int_{T} u^{\prime}(t) w^{\prime}(t) d t+\int_{T} u(t) w(t) d t=\int_{T} h(t) w(t) d t, \quad \forall w \in C_{c}(] 0, b[) .
$$

Hence, $u \in W^{2,2}(T, H)$ and $u$ is a solution of problem (4).

Proposition 4: The operator $(I+\widehat{L})^{-1}: L^{2}(T, H) \rightarrow D \subseteq W^{1,2}(T, H)$ is completely continuous.

Proof: Let $\left\{x_{n}\right\}_{n}$ be a sequence in $L^{2}(T, H)$ such that $x_{n} \rightarrow x$ weakly and let $\left\{y_{n}\right\}_{n} \subset D$ be the sequence $y_{n}=(I+\widehat{L})^{-1}\left(x_{n}\right), \forall n \in \mathbb{N}$. Since $\widehat{L}$ is a maximal monotone operator, it follows that $\left\|y_{n}\right\| \leq\left\|x_{n}\right\|, \forall n \in \mathbb{N}$ (cf. [3, Proposition 3.2]) and so $\left\{y_{n}\right\}_{n}$ is bounded in $L^{2}(T, H)$. Moreover, since $x_{n}=y_{n}-y_{n}^{\prime \prime}$, we have that also $\left\{y_{n}^{\prime \prime}\right\}_{n}$ is bounded in $L^{2}(T, H)$, therefore the sequence $\left\{y_{n}\right\}_{n}$ is bounded in $W^{2,2}(T, H)$. Since the latter embeds compactly in $W^{1,2}(T, H)$, by passing to a subsequence if necessary, we deduce that $y_{n} \rightarrow y$ in $W^{1,2}(T, H)$.

Now, taking into account that the operator $I+\widehat{L}$ is maximal monotone (cf. [3, Theorem 1.7]) we have that (cf. Remark 1) $G r(I+\widehat{L})$ is sequentially closed in $L^{2}(T, H) \times L^{2}(T, H)_{w}$. Here, $L^{2}(T, H)_{w}$ denotes the space $L^{2}(T, H)$ endowed with the weak topology. We conclude that $y=(I+\widehat{L})(x)$, and hence the operator $(I+\widehat{L})^{-1}$ is completely continuous.

Now we give the definition of "tube solution" to the problem (4), introduced in $[20]$ in the case of finite dimensional spaces. We extend this definition in a natural way to Hilbert spaces.

A couple of functions $(\sigma, M) \in W^{2,2}(T, H) \times W^{2,2}(T,[0,+\infty))$ is said to be a tube solution to problem (4) if

(i) for a.e. $t$ in $\{t \in T: M(t)>0\}$ and for every $(x, y) \in H \times H$ such that

$$
\|x-\sigma(t)\|=M(t) \text { and }\left\langle x-\sigma(t), y-\sigma^{\prime}(t)\right\rangle=M(t) M^{\prime}(t)
$$

there exists $v \in-F(t, x, y)$ such that

$$
\left\langle x-\sigma(t), v-\sigma^{\prime \prime}(t)\right\rangle+\left\|y-\sigma^{\prime}(t)\right\|^{2} \geq M(t) M^{\prime \prime}(t)+M^{\prime}(t)^{2}
$$

(ii) $\quad \sigma^{\prime \prime}(t) \in-F\left(t, \sigma(t), \sigma^{\prime}(t)\right)$ a.e. on $\{t \in T: M(t)=0\}$;

(iii) $\quad\left\|v_{0}-\sigma(0)\right\| \leq M(0),\left\|v_{1}-\sigma(b)\right\| \leq M(b)$.

Observe that requiring the existence of a tube solution for problem (4) is equivalent, in the scalar case, to requiring the existence of an upper and lower solution (cf. [20, p. 70]).

Using a similar proof to that of Lemma 5.10 of [20], we obtain the following preliminary result which we shall use in the existence theorem.

Lemma 5: Let $(\sigma, M)$ be a tube solution to problem (4) and $x \in W^{2,2}(T, H)$ be a function such that $x(0)=\nu_{0}$ and $x(b)=\nu_{1}$. If for almost every $t$ in $\{t \in T: \| x(t)-$ 
$\sigma(t) \|>M(t)\}$ it holds that $d\left(t, x(t), x^{\prime}(t), x^{\prime \prime}(t)\right) \geq M^{\prime \prime}(t)$, where

$$
d(t, x, y, v)=\frac{\left\langle x-\sigma(t), v-\sigma^{\prime \prime}(t)\right\rangle+\left\|y-\sigma^{\prime}(t)\right\|^{2}}{\|x-\sigma(t)\|}-\frac{\left\langle x-\sigma(t), y-\sigma^{\prime}(t)\right\rangle^{2}}{\|x-\sigma(t)\|^{3}},
$$

then $\|x(t)-\sigma(t)\| \leq M(t), \forall t \in T$.

Denote by $H_{1}$ the following condition:

$H_{1}$ : $\quad$ there exists a tube solution $(\sigma, M)$ for problem (4).

We have the following existence result.

Theorem 6: If hypotheses $\mathrm{H}_{1}$ and $\mathrm{H}(\mathrm{F})_{2}$ hold, then problem (4) has a solution such that $\|x(t)-\sigma(t)\| \leq M(t), \forall t \in T$.

Proof: First we introduce some functions that we shall use in the proof.

Let $u: T \times H \rightarrow H, \tilde{x}: T \times H \rightarrow H$ and $\widehat{y}: T \times H \times H \rightarrow H$ be functions defined by

$$
u(t, x)=\left\{\begin{array}{cc}
\frac{M(t)}{\|x-\sigma(t)\|}(\sigma(t)-x)+x-\sigma(t) & \text { if }\|x-\sigma(t)\|>M(t), \\
0 & \text { otherwise, }
\end{array}\right.
$$

$\forall(t, x) \in T \times H$

$$
\tilde{x}(t, x)=\left\{\begin{array}{cc}
\frac{M(t)}{\|x-\sigma(t)\|}(x-\sigma(t))+\sigma(t) & \text { if }\|x-\sigma(t)\|>M(t), \\
x & \text { otherwise, }
\end{array}\right.
$$

$\forall(t, x) \in T \times H$

$\widehat{y}(t, x, y)=$

$\left\{\begin{array}{cc}y+\left(M^{\prime}(t)-\frac{\left\langle x-\sigma(t), y-\sigma^{\prime}(t)\right\rangle}{\|x-\sigma(t)\|}\right)\left(\frac{x-\sigma(t)}{\|x-\sigma(t)\|}\right) & \text { if }\|x-\sigma(t)\|>M(t), \\ y & \text { otherwise, }\end{array}\right.$

$\forall(t, x, y) \in T \times H \times H$. Now let $\Gamma: T \times H \times H \rightarrow 2^{H}$ be the multifunction defined by

$\Gamma(t, x, y)=\left\{\begin{array}{cc}\left\{v \in H:\left\langle\widetilde{x}(t, x)-\sigma(t), v-\sigma^{\prime \prime}(t)\right\rangle+\left\|\widehat{y}(t, x, y)-\sigma^{\prime}(t)\right\|^{2} \geq M(t) M^{\prime \prime}(t)+M^{\prime}(t)^{2}\right\} \\ H & \text { if }\|x-\sigma(t)\|>M(t), \\ & \text { otherwise, }\end{array}\right.$

$\forall(t, x, y) \in T \times H \times H$ and let $\Phi: T \times H \times H \rightarrow 2^{H}$ be the multifunction

$$
\Phi(t, x, y)=\tilde{F}(t, x, y)+g(t, x), \forall(t, x, y) \in T \times H \times H
$$

where $g: T \times H \rightarrow H$ and $\tilde{F}: T \times H \times H \rightarrow 2^{H}$ are defined by 


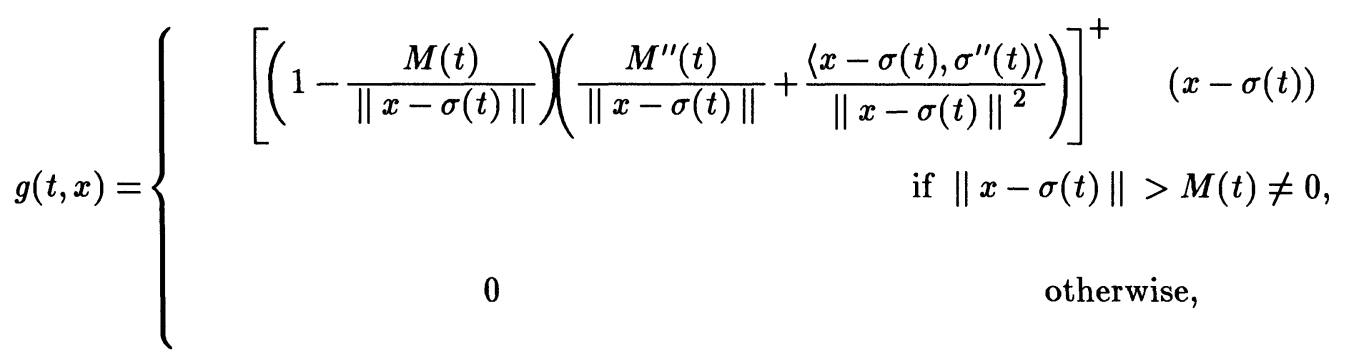

$\forall(t, x) \in T \times H$

$$
\widetilde{F}(t, x, y)=\left\{\begin{array}{cc}
\frac{M(t)}{\|x-\sigma(t)\|}(-F(t, \tilde{x}(t, x), \widehat{y}(t, x, y)) \cap \Gamma(t, x, y)) & \text { if }\|x-\sigma(t)\|>M(t)>0 \\
-F(t, x, y) & \text { if }\|x-\sigma(t)\| \leq M(t) \neq 0 \\
\sigma^{\prime \prime}(t) & \text { if } M(t)=0
\end{array}\right.
$$

$\forall(t, x) \in T \times H \times H$.

Observe that $\forall(t, x, y) \in T \times H \times H$ we have that $\Phi(t, x, y) \neq \emptyset$. This is evident when $\|x-\sigma(t)\| \leq M(t) \neq 0$ or $M(t)=0$. If $\|x-\sigma(t)\|>M(T)>0$, then $\|\tilde{x}(t, x)-\sigma(t)\|=M(t)$ and $\left\langle\tilde{x}(t, x)-\sigma(t), \widehat{y}(t, x, y)-\sigma^{\prime}(t)\right\rangle=M(t) M^{\prime}(t)$. Therefore from $H_{1}$ we deduce the existence of an element $v \in-F(t, \tilde{x}(t, x), \widehat{y}(t, x, y))$ with the property that $\left\langle\tilde{x}(t, x)-\sigma(t), v-\sigma^{\prime \prime}(t)\right\rangle+\left\|\widehat{y}(t, x, y)-\sigma^{\prime}(t)\right\|^{2} \geq M(t) M^{\prime \prime}(t)+$ $M^{\prime}(t)^{2}$ and hence $v \in \widetilde{F}(t, x, y)$.

On the other hand, using the properties of $F$ and observing that $\Gamma$ has closed and convex values, we infer that $\Phi$ is a multifunction with closed, convex and bounded values and it is integrably bounded in $L^{2}\left(T, \mathbb{R}^{+}\right)$, since $\|\tilde{x}(t, x)\| \leq M(t)+$ $\|\sigma(t)\|$ and $\|g(t, x)\| \leq\left|M^{\prime \prime}(t)\right|+\left\|\sigma^{\prime \prime}(t)\right\|$ a.e. on $T$.

We now consider the following Dirichlet boundary value problem

$$
\left\{\begin{array}{c}
x^{\prime \prime}(t) \in \Phi\left(t, x(t), x^{\prime}(t)\right)+u(t, x(t)) \quad \text { a.e. on } T \\
x(0)=v_{0}, x(b)=v_{1}
\end{array}\right.
$$

and put $S=\left\{x \in W^{2,2}(T, H): x\right.$ is a solution of $\left.(6)\right\}$.

Suppose initially that $S \neq \emptyset$. We shall prove that every element of $S$ satisfies $\|x(t)-\sigma(t)\| \leq M(t)$ in $T$. We will then prove that $S \neq \emptyset$ and hence every element of $S$ is a solution of our problem (4).

Claim 1: $x \in S \Rightarrow\|x(t)-\sigma(t)\| \leq M(t), \forall t \in T$.

First observe that denoting by $T^{\prime}$ and $T^{\prime \prime}$ respectively, the sets $T^{\prime}=\{t \in T$ : $\left.M(t)=0, M^{\prime}(t) \neq 0\right\}$ and $T^{\prime \prime}=\left\{t \in T: M^{\prime}(t)=0, M^{\prime \prime}(t) \neq 0\right\}$, from the Banach Lemma (cf. [20, Lemma A.9]) it follows that $m T^{\prime}=m T^{\prime \prime}=0$. Fix $t \in T \backslash\left(T^{\prime} \cup T^{\prime \prime}\right.$ ) and $(x, y) \in H \times H$ such that $\|x-\sigma(t)\|>M(t)$ and let $v$ be an element of $\Phi(t, x, y)+u(t, x)$.

In the case in which $M(t)>0$, we put $v=\frac{M(t)}{\|x-\sigma(t)\|} v_{1}+g(t, x)+u(t, x)$, where $v_{1}$ is an element of $\Gamma(t, x, y)$ and so it satisfies the following inequality 
$\left\langle\frac{M(t)(x-\sigma(t))}{\|x-\sigma(t)\|}, v_{1}-\sigma^{\prime \prime}(t)\right\rangle+\left\|y-\sigma^{\prime}(t)\right\|^{2}-\frac{\left\langle x-\sigma(t), y-\sigma^{\prime}(t)\right\rangle^{2}}{\|x-\sigma(t)\|^{2}} \geq M(t) M^{\prime \prime}(t)$

since $\left\|\widehat{y}(t, x, y)-\sigma^{\prime}(t)\right\|^{2}=\left\|y-\sigma^{\prime}(t)\right\|^{2}+M^{\prime}(t)^{2}-\frac{\left\langle x-\sigma(t), y-\sigma^{\prime}(t)\right\rangle^{2}}{\|x-\sigma(t)\|^{2}}$. Now, put

$$
\eta(t, x)=\left[\left(1-\frac{M(t)}{\|x-\sigma(t)\|}\right)\left(\frac{M^{\prime \prime}(t)}{\|x-\sigma(t)\|}+\frac{\left\langle x-\sigma(t), \sigma^{\prime \prime}(t)\right\rangle}{\|x-\sigma(t)\|^{2}}\right)\right]^{+} .
$$

We have (cf. Lemma 5)

$$
\begin{gathered}
d(t, x, y, v)= \\
\frac{\left(\left\langle x-\sigma(t), \frac{M(t)}{\|x-\sigma(t)\|_{1}} v_{1}+\eta(t, x)(x-\sigma(t))+u(t, x)-\sigma^{\prime \prime}(t)\right\rangle\right)}{\|x-\sigma(t)\|} \\
+\frac{\left\|y-\sigma^{\prime}(t)\right\|^{2}}{\|x-\sigma(t)\|}-\frac{\left\langle x-\sigma(t), y-\sigma^{\prime}(t)\right\rangle^{2}}{\|x-\sigma(t)\|^{3}}=\eta(t, x)\|x-\sigma(t)\|+\frac{M(t)\left\langle x-\sigma(t), v_{1}-\sigma^{\prime \prime}(t)\right\rangle}{\|x-\sigma(t)\|^{2}} \\
+\frac{M(t)-\|x-\sigma(t)\|}{\|x-\sigma(t)\|^{2}}\left\langle\sigma^{\prime \prime}(t), x-\sigma(t)\right\rangle+\frac{\left\|y-\sigma^{\prime}(t)\right\|^{2}}{\|x-\sigma(t)\|}+\frac{\left\langle x-\sigma(t), y-\sigma^{\prime}(t)\right\rangle^{2}}{\|x-\sigma(t)\|^{3}} \\
+\frac{\langle x-\sigma(t), u(t, x)\rangle}{\|x-\sigma(t)\|} ;
\end{gathered}
$$

from which, by (4.2) we deduce that

$$
\begin{gathered}
d(t, x, y, v) \geq \eta(t, x)\|x-\sigma(t)\|+\frac{M(t)-\|x-\sigma(t)\|}{\|x-\sigma(t)\|^{2}}\left\langle\sigma^{\prime \prime}(t), x-\sigma(t)\right\rangle+\frac{M(t) M^{\prime \prime}(t)}{\|x-\sigma(t)\|} \\
+\frac{\langle x-\sigma(t), u(t, x)\rangle}{\|x-\sigma(t)\|} \geq\left(1-\frac{M(t)}{\|x-\sigma(t)\|}\right)\left(\frac{M^{\prime \prime}(t)}{\|x-\sigma(t)\|}+\frac{\left\langle x-\sigma(t), \sigma^{\prime \prime}(t)\right\rangle}{\|x-\sigma(t)\|^{2}}\right)\|x-\sigma(t)\| \\
+\frac{M(t)-\|x-\sigma(t)\|}{\|x-\sigma(t)\|}\left\langle\sigma^{\prime \prime}(t), x-\sigma(t)\right\rangle+\frac{M(t) M^{\prime \prime}(t)}{\|x-\sigma(t)\|}+\frac{\langle x-\sigma(t), u(t, x)\rangle}{\|x-\sigma(t)\|} \\
=M^{\prime \prime}(t)+\|x-\sigma(t)\|-M(t)
\end{gathered}
$$

and so $d(t, x, y, v) \geq M^{\prime \prime}(t)$.

In the case in which $M(t)=0$, since $v=\sigma^{\prime \prime}(t)+u(t, x(t))$, we have that

$$
\begin{gathered}
d(t, x, y, v)=\frac{\langle x-\sigma(t), u(t, x)\rangle}{\|x-\sigma(t)\|}+\frac{\left\|y-\sigma^{\prime}(t)\right\|^{2}}{\|x-\sigma(t)\|}-\frac{\left\langle x-\sigma(t), y-\sigma^{\prime}(t)\right\rangle^{2}}{\|x-\sigma(t)\|^{3}} \\
\geq\|x-\sigma(t)\| \geq 0
\end{gathered}
$$


Hence, since $t \notin T^{\prime} \cup T^{\prime \prime}$, we conclude that also in this case $d(t, x, y, v) \geq M^{\prime \prime}(t)$.

Now, if $x \in S$ we obtain that $d\left(t, x(t), x^{\prime}(t), x^{\prime \prime}(t)\right) \geq M^{\prime \prime}(t)$ a.e. on $\{t \in T$ : $\|x(t)-\sigma(t)\|>M(t)\}, \quad$ since $\quad x^{\prime \prime}(t) \in \Phi\left(t, x(t), x^{\prime}(t)\right)+u(t, x(t)) \quad$ a.e. $\quad$ on $T$. Therefore, from Lemma 5 , we conclude that $\|x(t)-\sigma(t)\| \leq M(t)$ for every $t \in T$ which proves the claim.

Claim 2: $S$ is nonempty.
Denote by $G: W^{1,2}(T, H) \rightarrow 2^{L^{2}(T, H)}$ the multifunction defined by

$$
G(x)=\left\{z \in L^{2}(T, H): z(t) \in \Phi\left(t, x(t), x^{\prime}(t)\right)+u(t, x(t))-x(t) \text { a.e. on } T\right\},
$$

$\forall x \in W^{1,2}(T, H)$. It is simple to check that the values of $G$ are nonempty, for every $x \in W^{1,2}(T, H)$. Now consider the operator $\Lambda: W^{1,2}(T, H) \rightarrow W^{1,2}(T, H)$, where (cf. Proposition 4)

$$
\Lambda(x)=-(I+\widehat{L})^{-1} G(x), \forall x \in W^{1,2}(T, H)
$$

First we observe that, since the values of $G$ are convex and $(I+\widehat{L})^{-1}$ is linear, the values of $\Lambda$ are convex and, moreover, from boundedness of the set $G\left(W^{1,2}(T, H)\right)$ and from Proposition 4, we obtain that the set $\Lambda(x)=-(I+\widehat{L})^{-1} G(x)$ is compact, $\forall x \in W^{1,2}(T, H)$.

We shall now prove that $\Lambda$ has a closed graph. To this end, from Proposition 4 and since $R(G)$ is bounded in $L^{2}(T, H)$, it is sufficient to prove that $G$ has a sequentially closed graph in $W^{1,2}(T, H) \times L^{2}(T, H)_{w}$. So denote by $G_{1}: W^{1,2}(T, H) \rightarrow$ $2^{L^{2}(T, H)}$ the Nemytskii operator associated to $\tilde{F}$, that is

$$
G_{1}(x)=\left\{z \in L^{2}(T, H): z(t) \in \widetilde{F}\left(t, x(t), x^{\prime}(t)\right) \text { a.e. on } T\right\}, \forall x \in W^{1,2}(T, H) .
$$

Since $\quad G(x)=G_{1}(x)+g(\cdot, x)+u(\cdot, x)-x$ and the function $\quad x \mapsto g(\cdot, x(\cdot))+$ $u(\cdot, x(\cdot))-x(\cdot)$ is continuous from $W^{1,2}(T, H)$ into $L^{2}(T, H)$, it is sufficient to prove that $G_{1}$ has a weakly sequentially closed graph.

Let $\left\{x_{n}\right\}_{n}$ be a sequence which converges to $x$ in $W^{1,2}(T, H)$ and $\left\{z_{n}\right\}_{n}$ be a sequence weakly convergent to $z$ in $L^{2}(T, H)$ such that $z_{n} \in G_{1}\left(x_{n}\right), \forall n \in \mathbb{N}$.

First we observe that it is possible to find a function $h \in L^{2}\left(T, \mathbb{R}^{+}\right)$and a subsequence of $\left\{x_{n}^{\prime}\right\}_{n}$, denoted again by $\left\{x_{n}^{\prime}\right\}_{n}$, such that $\forall x, y \in H$

$$
x_{n}^{\prime}(t) \rightarrow x^{\prime}(t),\|\tilde{F}(t, x, y)\|<h(t) \text { a.e. on } T .
$$

Applying Mazur's Theorem to the sequence $\left\{z_{n}\right\}_{n}$, we obtain that there exists a sequence $\left\{v_{n}\right\}_{n}, v_{n} \in \operatorname{co}\left\{z_{m}: m \geq n\right\}$ such that $v_{n} \rightarrow z$ in $L^{2}(T, H)$, and so by passing to a subsequence if necessary, we have that

$$
v_{n}(t) \rightarrow z(t) \text { a.e. on } T \text {. }
$$

On the other hand, since $\widetilde{T}=\{t \in T:\|x(t)-\sigma(t)\|>0\}$ is an open subset of $T$, we can represent it as $\widetilde{T}=\bigcup_{n}\left(a_{n}, b_{n}\right)$, where $\left(a_{n}, b_{n}\right) \subset T$. Therefore by the absolutely continuity of $t \rightarrow\|x(t)-\sigma(t)\|-M(t)$ and the Banach Lemma (cf. [20, Lemma A.9]) we obtain

$$
m\left(\bigcup_{n}\left\{t \in\left(a_{n}, b_{n}\right):\|(x-\sigma)(t)\|=M(t),\|(x-\sigma)(t)\|^{\prime} \neq M^{\prime}(t)\right\}\right)=0 .
$$


Now fix $t \in T$ such that it satisfies (4.3), (4.4) and

$$
t \notin \bigcup_{n}\left\{t \in\left(a_{n}, b_{n}\right):\|(x-\sigma)(t)\|=M(t),\|(x-\sigma)(t)\|^{\prime} \neq M^{\prime}(t)\right\} .
$$

First we consider the case in which $M(t)>0$ and $\|x(t)-\sigma(t)\|>M(t)$. Put $R=\{r \in H:\|r-\sigma(t)\|>M(t)\}$. Then there exists a closed ball $B$ with center $x(t)$ and $\bar{n} \in \mathbb{N}$ such that $x_{n}(t) \in B \subset \mathbb{R} \forall n>\bar{n}$. Observe that the multifunction $\left.\widetilde{F}\right|_{B \times H}$ has a weakly sequentially closed graph since the map $(x, y) \mapsto$ $F(t, \widetilde{x}(t, x), \widehat{y}(t, x, y))$ is (u.s.c.) in $B \times H$ and $\left.\Gamma\right|_{B \times H}$ has a weakly sequentially closed graph. From this we deduce that the multifunction $(x, y) \mapsto \widetilde{F}(t, x, y)$ is (u.s.c.) from $B \times H$ into $H_{w}$ and so, for a fixed neighborhood $U$ of zero in $H_{w}$, since $\widetilde{F}$ has convex values, we obtain that there exists $n_{U}>\bar{n}$ such that $v_{n} \in \widetilde{F}\left(t, x(t), x^{\prime}(t)\right)+U$, $\forall n>n_{U}$. From the arbitrary choice of $U$ and since $\widetilde{F}$ has closed values, we have that $z(t) \in G_{1}(x)(t)$.

In the case that $M(t)>0$ and $\|x(t)-\sigma(t)\|<M(t)$ we can find $\tilde{n} \in \mathbb{N}$ such that $\left\|x_{n}(t)-\sigma(t)\right\| \leq M(t), \forall n>\tilde{n}$ and so, from the upper semicontinuity of $(x, y) \mapsto$ $\widetilde{F}(t, x, y)$ we obtain again, $z(t) \in G_{1}(x)(t)$.

If $M(t)>0$ and $\|x(t)-\sigma(t)\|=M(t)$, since $x_{n}(t) \rightarrow x(t)$, we have that either there exists $n^{*} \in \mathbb{N}$ such that $\left\|x_{n}(t)-\sigma(t)\right\| \leq M(t), \forall n>n^{*}$ or there exists a subsequence of $\left\{x_{n}(t)\right\}_{n}$, denoted again by $\left\{x_{n}(t)\right\}_{n}$ such that $\left\|x_{n}(t)-\sigma(t)\right\|>M(t)$, $\forall n \in \mathbb{N}$. In the first case, proceeding as above, we obtain that $z(t) \in G_{1}(x)(t)$, while in the second case, we observe that from the upper semicontinuity of $(x, y) \mapsto$ $F(t, \tilde{x}(t, x), y)$ in $\left(x(t), x^{\prime}(t)\right)$ and since $\widehat{y}\left(t, x_{n}(t), x_{n}^{\prime}(t)\right) \rightarrow x^{\prime}(t)$, we have that for every neighborhood $V$ of zero in $H$, there exists $n_{*} \in \mathbb{N}$ such that

$$
\begin{gathered}
z_{n}(t) \in \frac{M(t)}{\left\|x_{n}(t)-\sigma(t)\right\|}\left(-F\left(t, \tilde{x}\left(t, x_{n}(t)\right), \widehat{y}\left(t, x_{n}(t), x_{n}^{\prime}(t)\right)\right) \cap \Gamma\left(t, x_{n}(t), x_{n}^{\prime}(t)\right)\right. \\
\subset-F\left(t, x(t), x^{\prime}(t)\right)+V \quad \forall n>n_{*},
\end{gathered}
$$

and so $z(t) \in G_{1}(x)(t)$. Finally, if $M(t)=0$, since $\tilde{F}\left(t, x_{n}(t), x_{n}^{\prime}(t)\right)=\tilde{F}(t, x(t)$, $\left.x^{\prime}(t)\right)=\sigma^{\prime \prime}(t)$, we deduce the desired property. Therefore $G_{1}$ has a weakly sequentially closed graph.

Now, since $\Lambda$ is a compact multifunction, we can apply the Kakutani-Ky-Fan fixed point theorem (cf. [20, Theorem A.2]) and conclude there exists $x \in W^{1,2}(T, H)$ such that

$$
\left\{\begin{array}{c}
x^{\prime \prime}(t) \in \Phi\left(t, x(t), x^{\prime}(t)\right)+u(t, x(t)) \quad \text { a.e. on } T \\
x(0)=v_{0}, x(b)=v_{1}
\end{array}\right.
$$

Now, from Claim 1, we have that $\|x(t)-\sigma(t)\| \leq M(t), \forall t \in T$, and so for a.e. $t$ in $T$, we have

$$
x^{\prime \prime}(t) \in \tilde{F}\left(t, x(t), x^{\prime}(t)\right)=\left\{\begin{array}{cc}
-F\left(t, x(t), x^{\prime}(t)\right) & \text { if } M(t) \neq 0 \\
\sigma^{\prime \prime}(t) & \text { if } M(t)=0 .
\end{array}\right.
$$

Applying Banach's Lemma (cf. [20, Lemma A.9]) and the definition of a tube solution we obtain that $x$ is indeed a solution of problem (4). 
Remark 3: Observe that, even if $H=\mathbb{R}^{N}$, Theorem 6 extends Theorem 5.9 of [20] in the sense that there exist multifunctions $F$ which satisfy our conditions but not those of the result of [20].

An example is proved by the following multifunction $F:[0,1] \times \mathbb{R}^{2} \times \mathbb{R}^{2} \rightarrow 2^{\mathbb{R}^{2}}$ defined by

$$
F(t, x, y)=\left\{\begin{array}{cc}
\left\{\left(\frac{1}{4 \sqrt{t}} \sin \|y\|, 0\right)\right\} & \text { if } t \neq 0 \\
\{(0,0)\} & \text { if } t=0 .
\end{array}\right.
$$

It is easy to see that $F$ satisfies the hypothesis $H(F)_{2}$ and $H_{1}$ by assuming $\sigma(t)=$ $(0,0)$ and $M(t)=1, \forall t \in T$.

On the other hand, $F$ does not satisfy condition (5.9.3) of Theorem 5.9 of [20].

The proof of Theorem 6 can be adapted (with minor modifications) to prove a similar existence result for the following periodic problem

$$
\left\{\begin{array}{l}
-x^{\prime \prime}(t) \in F\left(t, x(t), x^{\prime}(t)\right) \\
x(0)=x(b), x^{\prime}(0)=x^{\prime}(b)
\end{array} \quad \text { a.e. on } T\right.
$$

where $F: T \times H \times H \rightarrow P_{w k c}(H)$ is a multifunction.

Obviously we have that a couple of functions $(\sigma, M) \in W^{2,2}(T, H) \times$ $W^{2,2}(T,[0,+\infty))$ is said to be a tube solution to the problem (5) if

(i) For almost every $t$ in $\{t \in T: M(t)>0\}$ and for every $(x, y) \in H \times H$ such that

$$
\|x-\sigma(t)\|=M(t) \text { and }\left\langle x-\sigma(t), y-\sigma^{\prime}(t)\right\rangle=M(t) M^{\prime}(t)
$$

there exists $v \in-F(t, x, y)$ such that

$$
\left\langle x-\sigma(t), v-\sigma^{\prime \prime}(t)\right\rangle+\left\|y-\sigma^{\prime}(t)\right\|^{2} \geq M(t) M^{\prime \prime}(t)+M^{\prime}(t)^{2} ;
$$

(ii) $\quad \sigma^{\prime \prime}(t) \in-F\left(t, \sigma(t), \sigma^{\prime}(t)\right)$ a.e. on $\{t \in T: M(t)=0\}$;

(iii) $\quad \sigma(0)=\sigma(b),\left\|\sigma^{\prime}(0)-\sigma^{\prime}(b)\right\| \leq M^{\prime}(b)-M^{\prime}(0)$ and $M(0)=M(b)$.

Therefore we have the following result.

Theorem 7: If hypotheses $\mathrm{H}_{1}$ and $\mathrm{H}(\mathrm{F})_{2}$ hold, then problem (5) has a solution such that $\|x(t)-\sigma(t)\| \leq M(t), \forall t \in T$.

\section{References}

[1] Adams, R.A., Sobolev Space, Academic Press, New York 1975.

[2] Aubin, J.P. and Cellina, A., Differential Inclusion, Springer-Verlag, Berlin 1984.

[3] Barbu, V., Nonlinear Semigroups and Differential Equations in Banach Spaces, Noordhoff International Publ., Leyden, The Netherlands 1976.

[4] Berge, C., Espaces Topologiques, Functions, Multivoques, Dunod, Paris 1958.

[5] Bernfeld, S.R. and Lakshmikantham, V., An Introduction to Nonlinear Boundary Value Problems, Academic Press, New York 1974. 
[6] Brezis, H., Analisi Funzionale, Teoria ed Applicazioni, Liguori 1986.

[7] Cabada, A. and Nieto, J.J., Extremal solutions of second order nonlinear periodic boundary value problems, Appl. Math. Comp. 40 (1990), 135-145.

[8] Castaing, C. and Valadier, M., Convex Analysis and Measurable Multifunctions, Lecture Notes in Math 580, Springer-Verlag, New York 1977.

[9] Datko, R., On the integration of set-valued mappings in a Banach space, Fund. Math. 78 (1973), 205-208.

[10] Deimling, K., Multivalued Differential Equations, Walter de Gruyter, Berlin 1992.

[11] Dunford, N. and Schwartz, J., Linear Operator I, Wiley, New York 1958.

[12] Erbe, L.H., Nonlinear boundary value problems for second differential equations, J. Differ. Eqns. 7 (1970), 459-472.

[13] Erbe, L.H., Existence of solutions to boundary value problems for second order differential equations, Nonl. Analy. 6 (1982), 1155-1162.

[14] Erbe, L.H. and Krawcewicz, W., Nonlinear boundary value problems for differential inclusions $y^{\prime \prime} \in F\left(t, y, y^{\prime}\right)$, Ann. Polon. Math. LIV 3 (1991), 195-226.

[15] Fabry, C.H. and Habets, P., Upper and lower solutions for second order boundary value problems with nonlinear boundary conditions, Nonl. Analy. 10 (1986), 985-1007.

[16] Frigon, M., Application de la Théorie de la Transversalité Topologique des Problèmes non Linéaires pour des équations Différentialles Ordinairès, Dissertationes Math 1990.

[17] Frigon, M., Problèmes aux limites pour des inclusions différentielles sans condition de croissance, Ann. Polon. Math. LIV 1 (1991), 69-83.

[18] Frigon, M., Théorèmes d'existence pour des problèmes aux limites sans condition de croissance, Bull. Sc. Math $2^{e}$ Series 117 (1993), 377-400.

[19] Frigon, M., Boundary and periodic value problems for system of differential equations under Bernstein-Nagumo growth condition, Diff. and Integ. Eqns. 8:7 (1995), 1789-1804.

[20] Frigon, M., Théorèmes d'existence de solutions d'inclusions différentialles, Topl. Meth. in Differ. Eqns. and Inclus. (1995), 51-87.

[21] Frigon, M. and Lee, J.W., Existence principles for Carathéodory differential equations in Banach spaces, Topol. Meth. Nonl. Analy. 1 (1993), 95-111.

[22] Fucik, S., Solvability of Nonlinear Equations and Boundary Value Problems, D. Reidel, Hingham 1980.

[23] Gaines, R., A priori bounds for solutions to nonlinear second order boundary value problems, Appl. Analy. 3 (1973), 157-167.

[24] Gaines, R. and Mawhin, J., Confidence Degree and Nonlinear Differential Equations, Lect. Notes in Math. 568, Springer-Verlag, Berlin 1977.

[25] Gao, W. and Wang, J., On a nonlinear second order periodic boundary value problem with Carathéodory functions, Ann. Polon. Math. LXII (1995), 238-291.

[26] Gaprindashvili, G.D., Solvability of a Dirichlet boundary value problem for system of nonlinear ordinary differential equations with singularities, Differ. Eqns. 27 (1991), 1074-1077.

[27] Gilbarg, D. and Trudinger, N., Elliptic Partial Differential Equations of Second Order, Springer-Verlag, New York 1977.

[28] Granas, A. and Guennon, Z.E., Quelques résultates dans lat théorie de Bernstein-Carathéodory de l'equation $y^{\prime \prime} \in f\left(t, y, y^{\prime}\right), C$.R. Acad. Sci. Paris Sér. I. Math. 306 (1988), 703-706. 
[29] Granas, A., Guenther, R.B. and Lee, J.W., Nonlinear Boundary Value Problems for Ordinary Differential Equations, Dissertationes Math. 1985.

[30] Granas, A., Guenther, R.B. and Lee, J.W., Some existence results for the differential inclusions $y^{(k)} \in F\left(x, y, \ldots, y^{(k-1)}\right), y \in B$, C.R. Acad. Sci. Paris Sér I Math. 307 (1988), 391-396.

[31] Habets, P. and Schmitt, K., Nonlinear boundary value problems for system of differential equations, Arch. Math. (Basel) 40 (1983), 441-446.

[32] Himmelberg, C.J., Measurable relations, Fund. Math. (1975), 53-73.

[33] Jackson, L.K., Subfunctions and second order differential inequalities, Adv. in Math. 2 (1968), 307-363.

[34] Jackson, L.K., Boundary value problems for ordinary differential equations, In: Studies in Ordinary Differential Equations (ed. by J.K. Hale), MAA Studies in Math 14, Math Assoc. Amer., Washington, D.C. (1977).

[35] Mawhin, J., Compacité monotonie et convexité dans l'étude de problémes aux limites semi-linéaires, Séminaire d'Analyse Moderne 19 (1981).

[36] Nagumo, M., Über die Differentialgleichung $y^{\prime \prime}=f\left(t, y, y^{\prime}\right)$, Proc. Phys. Math. Soc. Japan 19:3 (1937), 861-866.

[37] Omari, P., A monotone method for constructing extremal solutions of second order scalar boundary value problem, Appl. Math. Comp. 28 (1986), 257-275.

[38] Papageorgiou, N.S. and Papalini, F., Periodic and boundary value problems for second order differential equations, (to appear).

[39] Perron, O, Eine neue Behandlung der Randwertaufgabe für $\Delta u=0$, Math. Z. 18 (1923), 42-54.

[40] Pruszko, T., Topological degree methods in multivalued boundary value problems, Nonl. Analy. 5 (1981), 953-973.

[41] Pruszko, T., Some Applications of Topological Degree Theory to Multivalued Boundary Value Problems, Dissertationes Math. 1984.

[42] Schmitt, K. and Thompson, R., Boundary value problems for infinite systems of second order differential equations, J. Differ. Eqns. 18 (1975), 277-298.

[43] Taylor, A.E. and Lay, D.C., Introduction to Functional Analysis, Krieger Publ. Co., Malabar, Florida 1980.

[44] Tyn Myint, U., Ordinary Differential Equations, North Holland, New York 1978.

[45] Vinti, C., Teoria della Integrazione, Galeno Editrice, Perugia 1988.

[46] Zeidler, E., Nonlinear Functional Analysis and its Applications, SpringerVerlag, New York 1985. 


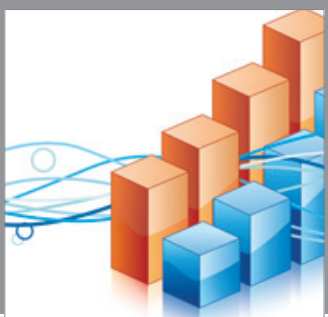

Advances in

Operations Research

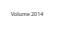

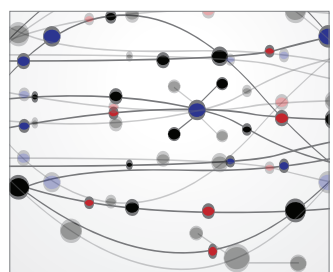

\section{The Scientific} World Journal
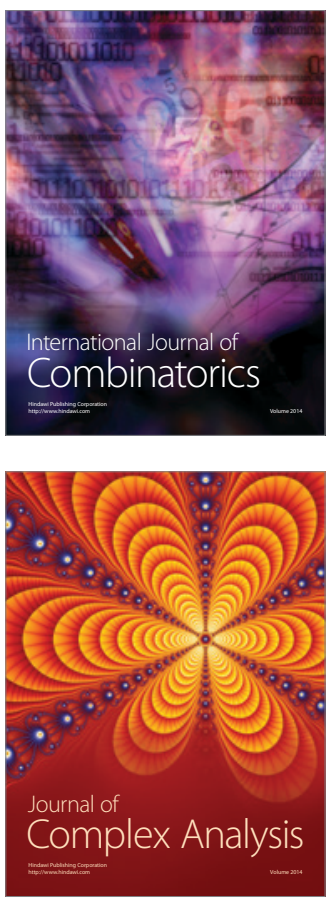

International Journal of

Mathematics and

Mathematical

Sciences
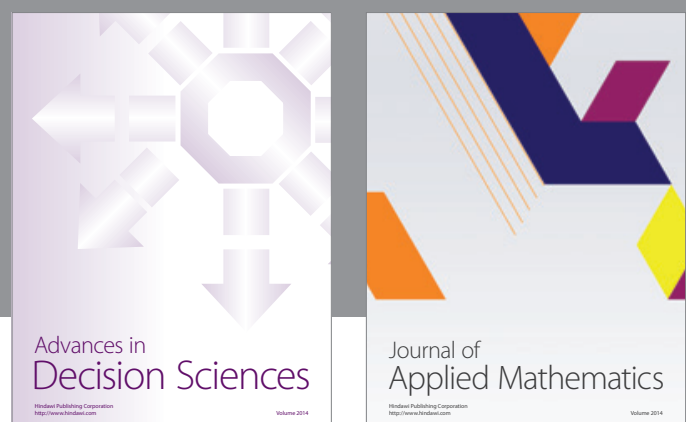

Journal of

Applied Mathematics
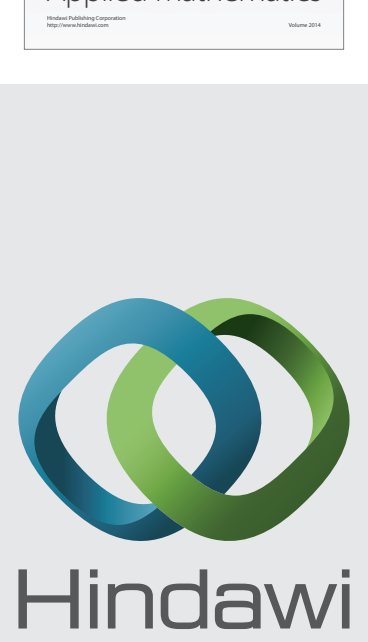

Submit your manuscripts at http://www.hindawi.com
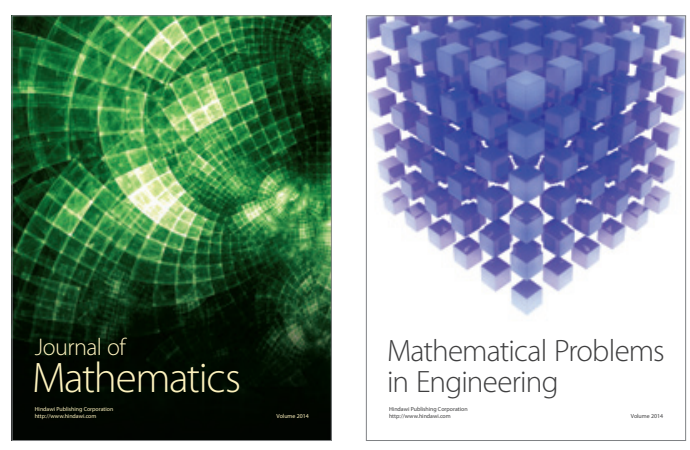

Mathematical Problems in Engineering
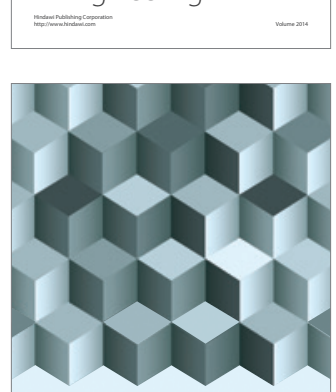

Journal of

Function Spaces
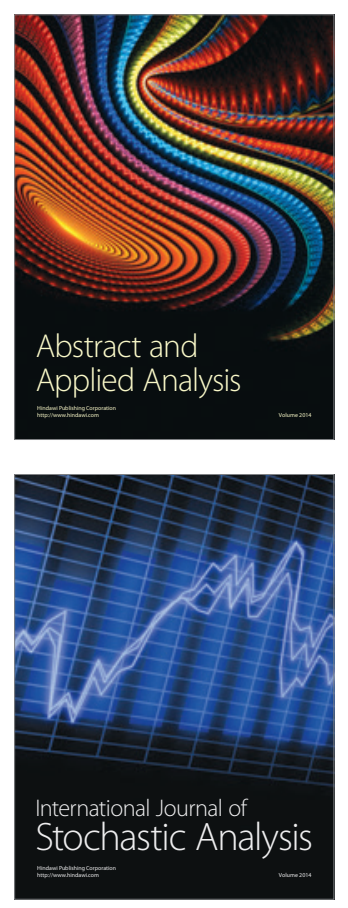

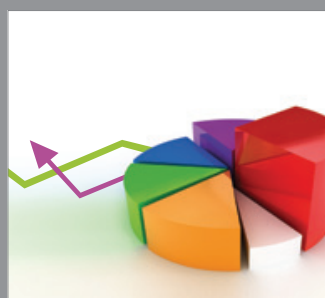

ournal of

Probability and Statistics

Promensencen
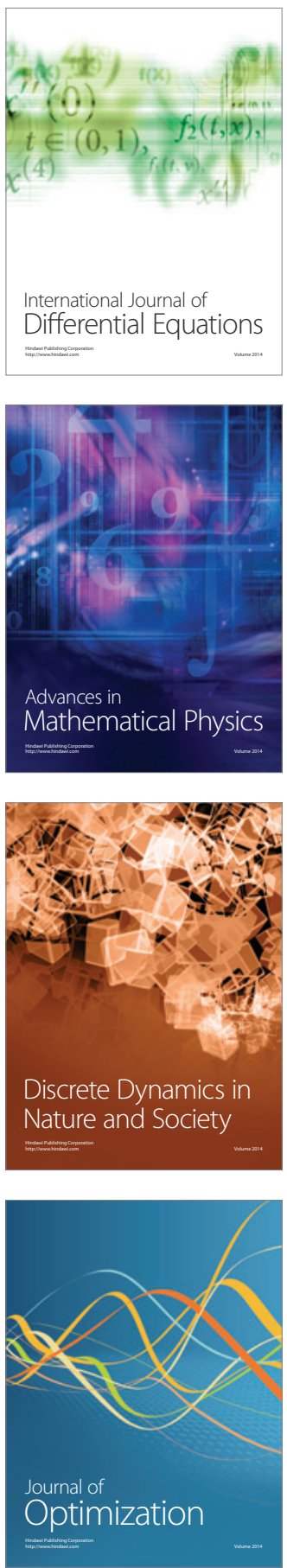